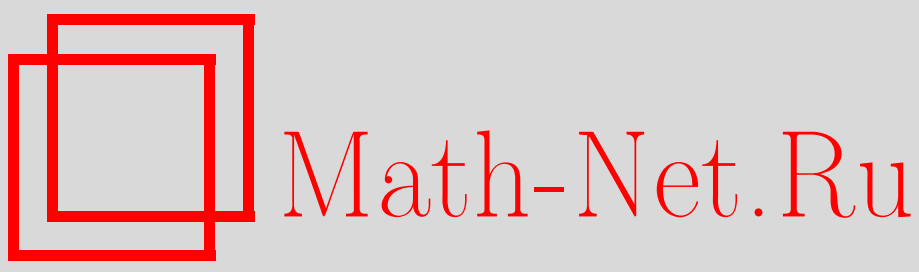

А. О. Иванов, А. А. Тужилин, Пространство взаимно параллельных линейных сетей с фиксированной границей, Изв. РАН. Сер. матем., 1999, том 63, выпуск 5, 83126

DOI: https://doi.org/10.4213/im260

Использование Общероссийского математического портала Math-Net.Ru подразумевает, что вы прочитали и согласны с пользовательским соглашением

http://www.mathnet.ru/rus/agreement

Параметры загрузки:

IP : 54.147 .182 .235

26 апреля 2023 г., 15:33:44 
УДК $514.77+512.816 .4+517.924 .8$

А.О. Иванов, А.А. Тужилин

\section{Пространство взаимно параллельных линейных сетей с фиксированной границей}

В данной работе изучается структура множества $[G, \varphi]_{\Gamma}$ погруженных линейных сетей в пространстве $\mathbb{R}^{N}$, параллельных заданной погруженной линейной сети $\Gamma: G \rightarrow \mathbb{R}^{N}$ и имеющих ту же границу $\varphi$, что и $\Gamma$. Доказывается, что $[G, \varphi]_{\Gamma}$ представляет собой выпуклое многогранное подмножество конфигурационного пространства подвижных вершин графа $G$. Кроме того, у этого выпуклого подмножества вычисляется размерность и оценивается количество граней максимальной размерности. Полученные результаты используются для описания пространства всех локально минимальных (взвешенных минимальных) сетей в пространстве $\mathbb{R}^{N}$ с фиксированными топологией и границей. Для случая плоских сетей, степени вершин которых не превосходят трех (сетей Штейнера), указанная размерность вычисляется в топологических терминах.

Библиография: 53 наименования.

\section{$\S 1$. Введение}

В настоящей статье изучаются семейства взаимно параллельных линейных сетей в евклидовом пространстве $\mathbb{R}^{N}$, у которых предполагаются фиксированными топология и гранища. Полученные результаты используются для описания пространств важных классов линейных сетей: локально минимальных и взвешенных минимальных сетей в $\mathbb{R}^{N}$ с фиксированной топологией и границей (оказывается, такие сети взаимно параллельны). Эти два класса сетей возникают как естественное обобшение плоских локально минимальных сетей, появляющихся при решении знаменитой проблемы Штейнера: из всех сетей (связных одномерных континуумов), затягивающих данное конечное множество точек плоскости, выбрать сеть наименьшей длины.

В теории минимальных сетей естественно выделяются так называемые сети Штейнера - сети, степени вершин которых не превосходят трех. Именно такие сети возникают как решения проблемы Штейнера (см. [14], [39], [17], [21], [23], [31]-[34], [44]). В работе [47] авторы изучили структуру множества плоских локально минимальных сетей Штейнера с фиксированньми топологией и гранищей. Было показано, что все такие сети взаимно параллельны и их множество представляет собой выпукльй многогранник в конфигурационном пространстве подвижных вершин параметризуюшего графа.

При работе над данной статьей авторы пользовались частичной поддержкой Российского фонда фундаментальных исследований (гранты № 98-01-00240 и № 96-15-96142), Международной Ассоциации по содействию сотрудничества с учеными Независимых Государств бывшего Советского Союза (грант № 97-0808). Авторы очень признательны этим организациям. 
В настоящей работе нами обнаружено, что в основе результатов из [47] лежит ряд обших фактов, касаюшихся устройства пространства всех взаимно параллельных линейных сетей с данной границей. Оказывается, уже это множество имеет структуру выпуклого многогранного множества (возможно, неограниченного), размерность которого может быть вычислена в терминах ранга некоторой линейной системы. В случае плоских локально минимальных сетей в работе [47] размерность соответствуюшего многогранника была вычислена в терминах топологии сети. Мы покажем, что аналогичный результат имеет место и для плоских взвешенных минимальных сетей Штейнера.

Отметим, что одной из хорошо известных интерпретаций проблемы Штейнера является транспортная проблема, состоящая в построении оптимальной (в смысле стоимости, а значит, и длины) сети дорог, соединяющих данную систему пунктов. При классической интерпретации все рассматриваемые дороги считаются равнозначными, т.е. стоимость участка дороги не зависит от его расположения в системе. Это приводит к тому, что целевой функционал пропорционален сумме длин всех дорог, входящих в систему. Однако часто оказывается, что стоимость разных дорог в системе по-разному зависит от их длины. Например, такое возможно, если нужно строить дороги разной ширины или работы выполняются разными фирмами и т.д. Последнее соображение приводит к тому, что в качестве целевой функции необходимо рассматривать функционал взвешенной длинь, т.е. линейную комбинацию длин дорог с некоторыми положительными постоянными коэффициентами. Сети, минимизирующие функционалы такого типа, и называются взвешенными минимальными сетями.

Сформулируем теперь основные результаты данной работы.

Пусть $G$ - произвольньй связньй граф, представленньй в виде одномерного конечного клеточного комплекса, и $\partial G$ - некоторая его граница (т.е. подмножество множества вершин графа $G$ ). Обобщенной сетью типа $G$ на многообразии $W$ называется непрерывное отображение Г графа $G$ в $W$, а границей $\partial \Gamma$ сети $\Gamma$ - ограничение отображения $\Gamma$ на гранищу $\partial G$ графа $G$. Графф $G$ в этом случае назьвается

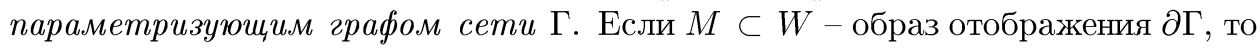
говорят, что сеть $Г$ затягивает множсество $M$. Ограничения отображения Г на вершины и ребра графа $G$ называются соответственно вериинами и ребрами сети Г. Вершины графа (соответствующие вершины сети), принадлежашие гранище, называются граничнылми (или неподвижнылми), а все остальные вершины внутренними (или подвижными). Обобщенную сеть Г будем называть погруженной, если все ее ребра - регулярные кривые.

Пусть фиксированы граф $G$ с гранищей $\partial G$ и некоторое граничное отображение $\varphi: \partial G \rightarrow W$. Рассмотрим всевозможные сети типа $G$ с границей $\varphi$. Положение граничных вершин этих сетей однозначно задается граничным отображением $\varphi$. Пространство всевозможных расположений подвижных вершин сетей типа $G$ с границей $\varphi$ назовем конфигурационным пространством подвижных вериин графа $G$ (по отношению $\kappa \varphi$ ). Отметим, что если многообразие $W$ связно, то это пространство представляет собой прямое произведение $s$ экземпляров $W$, где $s-$ количество подвижных вершин графа $G$. Если же $W$ не связно, то в силу связности графа $G$ конфигурационное пространство подвижных его вершин совпадает с прямым произведением $s$ экземпляров связной компоненты $W_{c}$ многообразия $W$, в которой содержится образ граничного отображения $\varphi$. 
Обобшенную сеть $\Gamma: G \rightarrow \mathbb{R}^{N}$ назовем линейной, если все ее ребра - прямолинейные отрезки (возможно, вырожденные). Пусть Г и $\Gamma^{\prime}$ - две погруженные линейные сети одного и того же типа $G$. Ориентируем граф $G$ произвольным образом. Это позволит нам рассматривать образы ребер этого графа при отображениях $\Gamma$ и $\Gamma^{\prime}$ как векторы в пространстве $\mathbb{R}^{N}$. Мы скажем, что сети $\Gamma$ и $\Gamma^{\prime}$ параллельны, если для каждого ребра $e$ из $G$ векторы $\Gamma(e)$ и $\Gamma^{\prime}(e)$ сонаправлены.

Пусть задана погруженная линейная сеть $\Gamma: G \rightarrow \mathbb{R}^{N}$ типа $G$ с некоторой фиксированной границей $\varphi$. Основным результатом настояшей статьи является описание множества $[G, \varphi]_{\Gamma}$ всех параллельных $Г$ линейных сетей с той же границей $\varphi$. Приведем это описание.

Разрежем граф $G$ по всем граничным вершинам степени больше 1 и определим границу каждой связной компоненты $G_{i}$ как множество всех тех ее вершин степени 1 , которые порождены вершинами из $\partial G$. Полученное разбиение графа $G$ назовем разбиением на невырожденные компоненты $G_{i}$.

Рассмотрим следующую линейную систему уравнений. Фиксируем в пространстве $\mathbb{R}^{N}$ некоторые евклидовы координаты. Фиксируем некоторую ориентацию сети Г и будем называть эту ориентацию исходной. Если $e-$ произвольное ребро сети $\Gamma$, ориентированное одним из двух возможных способов, то положим $\varepsilon(e)$ равным 1 , если эта ориентация ребра $e$ совпадает с исходной. Иначе положим $\varepsilon(e)=-1$. Пусть $\nu(e)-$ координаты единичного вектора направления ребра $e$ сети Г в исходной ориентации.

Разобьем граф $G$ на невырожденные компоненты, и пусть $\Gamma_{i}=\left.\Gamma\right|_{G_{i}}-$ соответствуюшие компоненты сети $\Gamma$. Рассмотрим произвольную компоненту $\Gamma_{i}$. Обозначим через $M_{1}, \ldots, M_{n}$ координаты образов граничных вершин $v_{1}, \ldots, v_{n}$ сети $\Gamma_{i}$. Пусть $e_{j}$ - единственное граничное ребро, инцидентное вершине $v_{j}$. Для каждого ребра $e_{j}$, отличного от $e_{1}$, фиксируем некоторый ориентированный путь $\gamma_{j}$ в $\Gamma_{i}$, соединяюший ребра $e_{1}$ и $e_{j}$, начинаюшийся в $v_{1}$ и заканчиваюшийся в $v_{j}$. Отметим, что ориентация пути $\gamma_{j}$ определяет значения чисел $\varepsilon(e)$ для всех ребер $e$ из $\gamma_{j}$. Далее, пусть $c_{1}, \ldots, c_{k}$ - циклы из $\Gamma_{i}$, соответствуюшие некоторой фундаментальной системе циклов графа $G_{i}$, на каждом из которых фиксирована ориентация. Как и в случае путей $\gamma_{j}$, ориентация каждого цикла $c_{q}$ определяет значения чисел $\varepsilon(e)$ для всех ребер $e$ из $c_{q}$.

Каждому ребру $е$ сети $\Gamma_{i}$ поставим в соответствие вещественную переменную $\lambda_{e}$. Рассмотрим следующую линейную систему уравнений на вещественные переменные $\lambda_{e}$, записанную в векторной форме:

$$
\begin{array}{ll}
\sum_{e: e \in \gamma_{j}} \varepsilon(e) \nu(e) \lambda_{e}=M_{j}-M_{1} & \forall \gamma_{j}, \\
\sum_{e: e \in c_{q}} \varepsilon(e) \nu(e) \lambda_{e}=0 & \forall c_{q} .
\end{array}
$$

Эта система линейных уравнений называется фундаментальной системой невырожденной компоненты $\Gamma_{i}$, а ее ранг - рангом $\mathrm{rk} \Gamma_{i}$ компоненты $\Gamma_{i}$.

ОПРЕДЕЛЕнИЕ. Фундаментальной системой сети Г называется линейная система уравнений, составленная из фундаментальных систем всех невырожденных компонент сети Г. Рангом $\operatorname{rk}(\Gamma)$ сети Г называется ранг ее фундаментальной системы. 
Отметим, что определенная нами фундаментальная система сети Г зависит от выбора путей $\gamma_{j}$ и циклов $c_{q}$. Оказывается, однако, что все фундаментальные системы сети Г эквивалентны как линейные системы уравнений. В частности, множества решений всех фундаментальных систем совпадают, и ранг сети Г определен корректно, т.е. не зависит от выбора фундаментальной системы сети.

ТЕОРема 1.1. Пусть $\Gamma$ - погруженная линейная сеть типа $G$ в $\mathbb{R}^{N}$ с границей $\varphi$. Обозначим через $r$ ранг сети $\Gamma$, а через $m$ - количество ребер сети Г. Тогда множество $[G, \varphi]_{\Gamma}$ погруженных линейных сетей типа $G c$ границей $\varphi$, параллельных $Г$, представляет собой (относительную) внутренность выпуклого $(m-r)$-мерного многогранного мнохсества (возмохно, не ограниченного) в конфигурационном пространстве подвижных вершин графа $G$. Более того, многогранное множество $[G, \varphi]_{\Gamma}$ содерхит не более чем $m$ граней максимальной размерности.

Из теоремы 1.1 вытекает ряд интересных следствий для важных классов линейных сетей: локально минимальных и взвешенных минимальных сетей. Напомним их определения.

Пусть $\Gamma: G \rightarrow W-$ произвольная погруженная сеть на многообразии $W$ и $P$ любая точка графа $G$. Замкнутую окрестность $U$ точки $P$ в $G$ назовем допустимой, если она не содержит вершин графа $G$, отличных от $P$, и не содержит петель из $G$. Каждая такая окрестность наделяется естественной структурой графа, который называется локальным графом с центром в точке $P$ и обозначается через $G_{U}$. Определим каноническую границу $\partial G_{U}$ локального графа $G_{U}$, положив $\partial G_{U}=(\partial G \cap U) \cup(G \cap \partial U)$. Сеть с гранищей, равная ограничению отображения Г на $G_{U}$, называется локальной сетью с иентром в точке $P$ и обозначается через $\Gamma_{U}$. При этом ограничение отображения $\Gamma$ на каноническую границу $\partial G_{U}$ локального графа $G_{U}$ называется канонической граничей локальной сети $\Gamma_{U}$ и обозначается через $\partial \Gamma_{U}$.

Пусть теперь $W$ - риманово многообразие. Погруженная сеть $Г$ с границей $\varphi$ называется абсолютно минимальной сетью с әраницей $\varphi$, если сеть Г имеет наименьшую длину среди всех сетей, затягивающих образ отображения $\varphi$. Погруженная сеть Г называется локально минимальной, если для каждой точки ее параметризуюшего графа существует абсолютно минимальная локальная сеть с центром в этой точке.

Перед тем как сформулировать следствие из теоремы 1.1, описывающее устройство пространства всех локально минимальных сетей данного типа с фиксированной границей, приведем следующее важное свойство таких сетей, обосновывающее применимость теоремы 1.1 в данном случае.

ПРЕДЛОЖЕНИЕ 1.1. Пусть $\Gamma_{1} u \Gamma_{2}$-две локально минимальные сети в $\mathbb{R}^{N}$ одного и того же типа и с одной и той же границей. Тогда сети $\Gamma_{i}-$ взаимно параллельные погруженные линейные сети.

СлЕДСТВИЕ 1.1. Пространство всех локально минимальных сетей в $\mathbb{R}^{N}$ данного типа с фиксированной границей или пусто, или является (относительной) внутренностью выпуклого многогранника в конфигурационном пространстве подвижных вершин графа $G$. B последнем случае размерность многогранника равна $m-r$, где $m$ - число ребер графа $G$, , $r$ - ранг фунда- 
ментальной системы любой из рассматриваемых сетей. Количество граней максимальной размерности этого многогранника не превосходит $\mathrm{m}$.

Пусть теперь $G$ - связньй взвешенный граф с гранищей $B$ и положительной весовой функцией $\omega$, заданной на множестве ребер графа $G$, а $W$ - некоторое риманово многообразие. Каждую сеть вида $\Gamma: G \rightarrow W$ назовем в этом случае взвешенной сетью типа $G$. Взвешенной длиной $\ell_{\omega}(\Gamma)$ взвешенной сети $\Gamma$ типа $G$ называется величина

$$
\ell_{\omega}(\Gamma)=\sum_{e \in E(\Gamma)} \omega(e) \ell(e)
$$

Отметим, что если $G$-взвешенный граф и $G_{U}-$ некоторый его локальный граф, то как на $G_{U}$, так и на соответствуюшей локальной сети $\Gamma_{U}$ естественным образом индуцируется весовая функция. Поэтому для каждой локальной сети взвешенной сети Г определена взвешенная длина.

Взвешенная сеть $\Gamma$ типа $G$, затягиваюшая конечное множество $M \subset W$ точек многообразия $W$, называется взвешенной минимальной сетью с границей $M$, если для любой ее точки $P$ некоторая локальная сеть $\Gamma_{U}$ с центром в $P$ имеет наименьшую взвешенную длину среди всех сетей типа $G_{U}$ с той же гранищей $\partial \Gamma_{U}$.

ЗАмечАниЕ. Отметим, что каждая локально минимальная сеть типа $G$ может быть рассмотрена как взвешенная минимальная сеть того же типа с постоянной весовой функцией. Поэтому, в частности, результаты настоящей работы, касающиеся плоских взвешенных минимальных сетей Штейнера, обобщают результаты из [47] о плоских локально минимальных сетях.

Чтобы использовать теорему 1.1 для описания множества всех взвешенных минимальных сетей с фиксированными топологией и границей, приведем аналог предложения 1.1.

ПРЕДЛОЖЕНИЕ 1.2. Пусть $\Gamma_{1} u \Gamma_{2}-$ две взвешенные минимальные сети в $\mathbb{R}^{N}$ одного и того же типа и с одной и той же границей. Тогда сети $\Gamma_{i}-$ взаимно параллельные погруженные линейные сети.

СлЕДСТВИЕ 1.2. Пространство всех взвешенных минимальных сетей в $\mathbb{R}^{N}$ данного типа с фиксированной граничей или пусто, или является (относительной) внутренностью выпуклого многогранника в конфигурационном пространстве подвижных вершин графа $G$. В последнем случае размерность многогранника равна $m-r$, где $m$ - число ребер графа $G$, а $r$ - ранг фундаментальной системы любой из рассматриваемых сетей. Количество граней максимальной размерности этого многогранника не превосходит $\mathrm{m}$.

Опишем теперь, чем могут отличаться друг от друга минимальные сети одного типа с фиксированной границей. Подвижныцм подграфом $\widehat{G}$ графа $G$ с границей $\partial G$ называется подграф, порожденньй множеством всех подвижных вершин графа $G$. Подвижную вершину погруженной линейной сети $\Gamma$ назовем слабо фuктивной, если все инцидентные ей ребра лежат на одной прямой. Слабо фиктивная вершина степени 2 называется просто фиктивной вершиной.

Разобьем все ребра погруженной линейной сети на уровни. Ребро сети $\Gamma: G \rightarrow \mathbb{R}^{N}$ отнесем к нулевому уровню, если оно принадлежит какому-нибудь циклу подвижного подграфа $\widehat{G}$ параметрического графа $G$ этой сети. Ребро, 
не принадлежашее нулевому уровню, но смежное с некоторым ребром нулевого уровня или инщидентное некоторой слабо фиктивной вершине сети, назовем ребром первого уровня, а все остальные ребра (т.е. ребра, не лежащие ни на нулевом, ни на первом уровнях) назовем ребрами второго уровня. Имеет место следующий результат.

ПРЕДЛОЖЕНИЕ 1.3. Любые две локально минимальные (взвешенные минимальные) сети одного и того же типа $G$ в $\mathbb{R}^{N}$ с границей $\varphi$ имеют одинаковую (взвешенную) длину и могут быть продеформировань друг в друга в классе локально минимальных (взвешенных минимальных) сетей того жее типа и с той же границей. Более того, при каждой такой деформачии остаются неподвижными все ребра второго уровня и все прямые, содержсаие ребра первого уровня.

Разобьем теперь вершины параметризуюшего графо $G$ на уровни. К нулевому уровню отнесем все (подвижные) вершины графа $G$, которые инцидентны только ребрам нулевого уровня. K первому уровню отнесем те подвижные вершины графа $G$, не попавшие на нулевой уровень, которые не инцидентны ребрам второго уровня. Все остальные вершины графа $G$ отнесем ко второму уровню. Для каждой вершины $v$ графа $G$ и каждого граничного отображения $\varphi$ назовем локусом вериины $v$ для отображения $\varphi$ замыкание подмножества пространства $\mathbb{R}^{N}$, состоящего из точек вида $\Gamma(v)$ по всем локально минимальным (взвешенньм минимальным) сетям $\Gamma$ типа $G$ с границей $\varphi$. Локус вершины $v$ для графа $G$ с границей $\varphi$ обозначим через $\operatorname{loc}_{\varphi}(v)$.

СлЕДСТВИЕ 1.3. В сделанных выше предположениях если $v$ - вериина второго уровня, то ее локус состоит из одной точки. Локус вериин первого и нулевого уровней представляет собой выпуклый многогранник в $\mathbb{R}^{N}$, размерность которого не превосходит $\min \{N, m-r\}$, а количество граней максимальной размерности не превосходит $m$.

Для плоских локально минимальных сетей и взвешенных минимальных сетей Штейнера число $(m-\mathrm{rk} \Gamma)$ и размерности локусов вершин сетей могут быть вычислены в геометрических терминах.

ТЕОРема 1.2. Пусть Г - или плоская локально минимальная сеть типа $G$ с гранищей $\varphi$, или не имеющая слабо фиктивных вершин плоская взвешенная минимальная сеть Штейнера типа $G$ с границей $\varphi$. Тогда множество локально минимальных сетей (взвешенных минимальных сетей Штейнера) типа $G$ с границей $\varphi$ представляет собой внутренность выпуклого $(k+f)$-мерного многогранника, где $k$ - иикломатическое число подвижного подграфа графа $G$, a $f$ - количество фиктивных вершин сети Г. Более того, этот многогранник содержит не более чем $m$ граней максимальной размерности, где $m$ - количество ребер сети Г.

Далее, локус $\operatorname{loc}_{\varphi}(v)$ произвольной вериины $v$ из $G$ имеет следующий вид. Если v-вершина второго уровня, то $\operatorname{loc}_{\varphi}(v)$ состоит из одной точки; если v из первого уровня, то $\operatorname{loc}_{\varphi}(v)$ - невырожденный прямолинейный отрезок; $u$ если $v$ - вериина нулевого уровня, то $\operatorname{loc}_{\varphi}(v)$ - выпуклый многоугольник, число вериин которого не превосходит $\mathrm{m}$.

Приведем здесь одно нетривиальное следствие этой теоремы. 
СлЕДСТВИЕ 1.4. В обозначениях теоремы 1.2 если $k=2 u f=0$, m.e. uикломатическое число подвижного подграфа графа $G$ равно 2 и сеть $\Gamma$ не содержит фиктивных вершин, то локусы всех вериин нулевого уровня выпуклье многоугольники с одинаковым числом сторон.

ЗАмечАНИЕ. Было бы интересно научиться вычислять размерность локусов вершин и число $\mathrm{rk} \Gamma$ для минимальных сетей в $\mathbb{R}^{N}$ общего вида в геометрических терминах.

Авторы, пользуясь случаем, выражают свою искреннюю и глубокую признательность академику РАН А. Т. Фоменко за его постоянное внимание к их работе.

\section{§ 2. Топологические графы}

Пусть $G$ - произвольное топологическое пространство, склеенное из конечного числа отрезков прямой $\left[a_{i}, b_{i}\right]$ по некоторой эквивалентности, отождествляюшей конщевые точки этих отрезков. Такое пространство $G$ называется топологическим графом. Если $\pi: \sqcup\left[a_{i}, b_{i}\right] \rightarrow G-$ проекция склейки, т.е. проекция, задаваемая этой склейкой, то образ каждого отрезка $\left[a_{i}, b_{i}\right]$ называется ребром топологического графа $G$, а образы точек $a_{i}$ и $b_{i}-$ вершинами из $G$. Сами отрезки $\left[a_{i}, b_{i}\right]$ назовем отрезками, параметризующими ребра топологического графа $G$. Множества вершин и ребер графа $G$ обозначим соответственно через $V(G)$ и $E(G)$.

Ясно, что мы получили топологическое представление абстрактных графов самого общего вида, т.е. графов с петлями и кратными ребрами, поэтому на топологические графы непосредственно переносится вся терминология как теории абстрактных графов, так и теории топологических пространств, чем мы и будем пользоваться в дальнейшем. Кроме того, в силу отмеченного соответствия между топологическими и абстрактными графами мы часто для краткости будем называть топологические графы просто графами, опуская слово “топологический”.

Предположим, что в графе $G$ выделено некоторое подмножество $B$ множества его вершин. Такой граф $G$ мы будем называть графом $c$ границей $\partial G=B$. Вершины из $\partial G$ будем называть граничнымми (или неподвижныьми), а все остальные вершины - внутренними (или подвижными). Ребра графа, инцидентные граничным вершинам, также назовем граничныцми, а ребро, не инцидентное никакой граничной вершине, назовем внутренним.

Пусть $G$ - некоторый граф с границей $B$. Обозначим через $\widehat{G}_{B}$ подграфф в $G$, порожденный всеми подвижными вершинами графа $G$. Подграф $\widehat{G}_{B}$ называется подвижныц подграфом графа $G$ (по отношению к границе $B$ ). Отметим, что подвижный подграф состоит в точности из всех внутренних ребер графа $G$.

Два топологических графа $G_{1}$ и $G_{2}$ называются эквивалентными, если между ними существует гомеоморфизм $\psi$, устанавливающий взаимно однозначное соответствие между множествами вершин этих графов. Каждый такой гомеоморфизм называется әквивалентностью. Если, кроме того, у графов $G_{i}$ имеются границы $B_{i}$, состояшие из одинакового числа вершин, и задано некоторое взаимно однозначное соответствие $\nu: B_{1} \rightarrow B_{2}$, то графы $G_{i}$ называются $\nu$-эквивалентнылми, если существует эквивалентность $\psi: G_{1} \rightarrow G_{2}$, ограничение которой на $B_{1}$ совпадает с $\nu$.

Пусть $G$ - произвольный граф с границей $\partial G$ (возможно, пустой) и $P \in G$ - некоторая его точка. Допустимой окрестностью $U \subset G$ точки $P$ графа $G$ называ- 
ется замыкание связной окрестности этой точки, не содержащее вершин графа $G$, отличных от $P$, если $P$ - вершина, и не содержашее петель из $G$. Наделим окрестность $U$ структурой графа, объявив вершинами все точки из $\partial U \cup(V(G) \cap U)$, а ребрами - отрезки в $U$, соединяюшие эти точки. Полученное дерево обозначим через $G_{U}$ и будем называть локальным графом с иентром в точке $P$. Определим каноническую границу $\partial G_{U}$ локального графа $G_{U}$, включив в нее все вершины из $\partial U$, а также точку $P$, если $P$ - граничная вершина графа $G$. Другими словами, $\partial G_{U}=(\partial G \cap U) \cup(G \cap \partial U)$.

Ниже мы используем локальные графы для определения взвешенных минимальных сетей. Здесь же проиллюстрируем, как работает это понятие, напомнив определение степени вершины. А именно, степенью вериины $v$ графа $G$ называется количество ребер произвольного локального графа с центром в $v$.

Пусть $G$ - топологический граф и $e$ - произвольное его ребро. Рассмотрим подпространство $G^{\prime}$ в $G$, полученное выкидыванием из $G$ внутренности ребра $e$. Наделим $G^{\prime}$ структурой топологического графа, объявив вершинами графа $G^{\prime}$ все вершины графа $G$, а ребрами графа $G^{\prime}$ - все ребра графа $G$, за исключением ребра $e$. Описанная только что перестройка графа $G$ называется выкидыванием из $G$ ребра е и обозначается $G \backslash e$.

Пусть $G$ - топологический граф и $v$ - произвольная его вершина. Пару $(G, v)$ назовем отмеченным топологическим графом.

Пусть $(G, v)$ и $\left(G^{\prime}, v^{\prime}\right)$ - два отмеченных топологических граффа. Пусть $I=$ $[a, b]$ - отрезок. Склеим $G, I$ и $G^{\prime}$ (как топологические пространства) следующим образом. Точку $a \in I$ отождествим с $v$, а точку $b \in I$ отождествим с $v^{\prime}$. Полученное топологическое пространство $\bar{G}$ наделим структурой граффа, выбрав в качестве вершин все вершины из $G$ и $G^{\prime}$, а в качестве ребер - все ребра из $G$ и $G^{\prime}$, а также отрезок $I$ (точнее, его образ при склейке). Эта операция называется склейкой отмеченных графов $(G, v) u\left(G^{\prime}, v^{\prime}\right)$, а ребро, полученное из отрезка $I,-$ ребром склейки.

Пусть $G$ - топологический графф и $v$ - произвольная его вершина степени больше 1. Построим новьй граф $G^{\prime}$, перестав отождествлять те конщы отрезков $\left[a_{i}, b_{i}\right]$, параметризуюших инцидентные $v$ ребра, которые склеиваются в вершину $v$ графа $G$. Говорят, что граф $G^{\prime}$ получен из $G$ разрезанием по вериине $v$. Ясно, что граф $G^{\prime}$ имеет столько же ребер, сколько и граф $G$, а количество вершин у графа $G^{\prime}$ больше, чем у графа $G$ на степень вершины $v$ без единицы. Если $\pi: \sqcup\left[a_{i}, b_{i}\right] \rightarrow G$ и $\pi^{\prime}: \sqcup\left[a_{i}, b_{i}\right] \rightarrow G^{\prime}-$ проекции склейки графов $G$ и $G^{\prime}$ соответственно, то, очевидно, существует единственное отображение $\nu: G^{\prime} \rightarrow G$ такое, что $\nu \circ \pi^{\prime}=\pi$. Это отображение переводит каждое ребро из $G^{\prime}$ в соответствующее ребро из $G$ и склеивает все вершины, полученные из вершины $v$. Отображение $\nu$ назовем восстанавливающим. Если граф $G^{\prime}$ получается из $G$ разрезанием по нескольким вершинам графа $G$, т.е. последовательным применением операции разрезания по вершине, то восстанавливающим отображением $\nu: G^{\prime} \rightarrow G$ в этом случае будем назьвать композищию восстанавливающих отображений.

Пусть $G$ - топологический граф̆, $e$ - произвольное его ребро и $I=[a, b]-$ отрезок, параметризующий ребро $e$. Выберем на $e$ (фактически на $[a, b]$ ) некоторую внутреннюю точку $A$ и добавим ее ко множеству вершин граффа $G$. При этом множество ребер графа $G$ перестроится следующим очевидным образом: ребро $e$ заменится на два ребра, инщидентных вершине $A$ степени 2. Разрежем теперь пере- 
строенный так граф по вершине $A$. Описанная операция называется разрезанием графа $G$ по точке $A$ и обозначается $G \backslash A$. Ребро $е$ будем называть ребром разреза, а точку $A$ - точкой разреза. Также скажем, что ребро е при разрезании по точке $A$ распадается на два ребра, а точка $A$ распадается на две вериины графа $G \backslash A$.

Определим теперь операцию на топологическом графе $G$, обратную к разрезанию. Для этого выберем в $G$ две вершины $v$ и $v^{\prime}$ степени 1 , и пусть $e$ и $e^{\prime}-$ ребра, инщидентные соответственно $v$ и $v^{\prime}$. Отождествим вершины $v$ и $v^{\prime}$. Полученное топологическое пространство обозначим через $G^{\prime}$. Параметризуем очевидньм образом объединение $e \cup e^{\prime}$ некоторым отрезком и после этого введем на $G^{\prime}$ структуру графа, выбрав в качестве вершин все вершины из $G$, за исключением $v$ и $v^{\prime}$, а в качестве множества ребер графа $G^{\prime}$ - множество всех ребер графа $G$, из которого выброшены $e$ и $e^{\prime}$ и добавлено $e \cup e^{\prime}$. Так полученный граф обозначим через $G / v \sim v^{\prime}$, а описанную операцию назовем склейкой графа $G$ по вершинам $v u v^{\prime}$. Вершины $v$ и $v^{\prime}$ называются вершинами склейки.

Пусть $G$ - некоторый графф с границей $\partial G$. Разрежем граф $G$ по всем граничным вершинам степени больше 1 и обозначим через $G_{i}$ полученные связные компоненты. Для каждой такой компоненты $G_{i}$ определим границу $\partial G_{i}$ как множество всех тех вершин из $G_{i}$ степени 1 , которые лежат в прообразе $\nu^{-1}(\partial G)$ границы $\partial G$ при восстанавливающем отображении $\nu$. Каждая компонента $G_{i}$ с границей $\partial G_{i}$ называется невырожденной компонентой графа $G$.

\section{§ 3. Обобщенные сети}

Пусть $G$ - связный граф. Непрерывное отображение Г графа $G$ в многообразие $W$ называется обобщенной сетью. При этом граф $G$ называется параметризующим графом сети $\Gamma$, или ее топологией, или ее типом. Ограничение отображения Г на вершины и ребра графа $G$ будем называть соответственно вериинами и ребрами сети Г. Ясно, что ребра сети - это непрерывные кривые на многообразии $W$. Отметим, что образы различных вершин графа $G$ при отображении $\Gamma$ могут, вообще говоря, совпадать. Будем обозначать через $V(\Gamma)$ и $E(\Gamma)$ соответственно множества вершин и ребер сети $Г$.

Выше мы определили различные операции над топологическими граффами. Во многих случаях они очевидным образом переносятся и на сети. Полученные в результате сети будем называть и обозначать так же, как и соответствующие топологические графы. Разберем теперь нетривиальные ситуации. К ним относятся склейка отмеченных графов и склейка графа по паре его вершин степени 1. В первом случае надо дополнительно определить сеть на ребре склейки, в чем заключается некоторый произвол. Ниже мы иногда будем накладывать дополнительные ограничения на ребра склейки таких сетей, что будет оговариваться особо. Во втором случае сеть может быть корректно определена, если и только если образы вершин склейки совпадают.

Пусть $\Gamma: G \rightarrow W-$ обобшенная сеть и $\partial G-$ граница графа $G$. Ограничение отображения $\Gamma$ на $\partial G$ называется границей сети $\Gamma$ и обозначается через $\partial \Gamma$. Если $M$ - произвольное конечное подмножество многообразия $W$ и $\Gamma$ - сеть с границей $\partial \Gamma$, образ которой совпадает с $M$, то будем говорить, что сеть $\Gamma$ затягивает множество $M$ по граничному отображению ӘГ. Вершины сети Г, принадлежащие границе $\partial Г$, называются граничными (или неподвижмными), а все остальные вершины из Г- внутренними (или подвижнылми). Ребра сети, инцидентные 
граничным вершинам, будем также называть граничными, а ребра, не инщидентные никакой граничной вершине, назовем внутренними.

Пусть $\Gamma: G \rightarrow W$ - обобшенная сеть и $\bar{\Gamma}: G \times[a, b] \rightarrow W$-непрерывное отображение. Обозначим через $\Gamma_{t}$ отображение $\bar{\Gamma}(\cdot, t)$. Тогда если $\Gamma_{a}=\Gamma$, то отображение $\bar{\Gamma}$ и семейство отображений $\Gamma_{t}$ называются деформаиией сети $\Gamma$.

Пусть теперь обобщенная сеть $\Gamma: G \rightarrow W$ имеет некоторую гранищу и $\Gamma_{t}-$ деформация сети Г. Мы говорим, что эта деформация неподвижна на әранице, если каждая граничная вершина сети $\Gamma$ неподвижна при деформации $\Gamma_{t}$.

Обобщенная сеть Г называется погруженной сетью, если каждое ее ребро регулярная кривая. Погруженная сеть Г называется вложенной, если отображение $\Gamma$ - топологическое вложение. Так как в дальнейшем нас будут интересовать преимущественно погруженные сети, то такие сети для краткости будем называть просто сетя.ми, опуская слово "погруженный".

\section{$\S 4$. Геодезические сети. Линейные сети}

Пусть $W$ - произвольное риманово многообразие.

ОПРЕДЕЛЕНИЕ. Обобшенная сеть в $W$, все ребра которой-отрезки геодезических (возможно, вырожденные), называется геодезической сетью.

В данном параграфе мы будем считать, что для многообразия $W$ выполнено следующее свойство: каждые две точки из $W$ соединяются единственной геодезической.

Пусть $\mathfrak{L}(G)$ - множество всех геодезических сетей вида $\Gamma: G \rightarrow W$, где $G-$ произвольный граф. Из введенного ограничения на $W$ вытекает, что элементы пространства $\mathfrak{L}(G)$ могут быть описаны положениями своих вершин. Поэтому если $|G|$ - количество вершин графа $G$, то $\mathfrak{L}(G)$ представимо в виде многообразия $W^{|G|}$. Это представление задает естественную гладкую структуру на $\mathfrak{L}(G)$, которая и будет иметься в виду в дальнейшем, когда мы будем говорить, например, о дифференщируемости функций, заданных на пространстве геодезических сетей. Элементы множества $\mathfrak{L}(G)$ назовем геодезическими сетями топологии $G$.

Пусть $G$ - граф с некоторой границей, а $\varphi$ - произвольное граничное отображение. Множество всех геодезических сетей $\Gamma: G \rightarrow W$ таких, что $\partial \Gamma=\varphi$, обозначим через $[G, \varphi]$ и будем называть пространством геодезических сетей типа $G$ c данной әраницей $\varphi$. По определению $[G, \varphi] \subset \mathfrak{L}(G)$. Каждый элемент из $[G, \varphi]$ однозначно задается положением своих подвижных вершин, поэтому если $s$ - количество подвижных вершин графа $G$, то $[G, \varphi]$ естественным образом наделяется структурой многообразия $W^{s} \subset W^{|G|}$. Многообразие $W^{s}=[G, \varphi]$ назьвается конфигурационным пространством подвижсных вершин графа $G$ (по отношению $к \varphi$ ).

В случае $W=\mathbb{R}^{N}$ геодезические сети называются линейными сетями. Случай линейных сетей представляет собой важный частный случай геодезических сетей. Для линейных сетей можно ввести понятие параллельности, которое оказывается чрезвычайно полезным при изучении геометрии локально минимальных и взвешенных локально минимальных сетей в евклидовом пространстве.

Пусть Г и $\Gamma^{\prime}$ - две погруженные линейные сети одного и того же типа $G$. Ориентируем граф $G$ произвольным образом. Это позволит нам рассматривать образы ребер этого граф̆а при отображениях $Г$ и $\Gamma^{\prime}$ как векторы в пространстве $\mathbb{R}^{N}$. Мы 
скажем, что сети $\Gamma$ и $\Gamma^{\prime}$ параллельнь, если для каждого ребра $e$ из $G$ векторы $\Gamma(e)$ и $\Gamma^{\prime}(e)$ сонаправлены.

Пусть $\Gamma: G \rightarrow \mathbb{R}^{N}$ - погруженная линейная сеть типа $G$ с произвольной фиксированной границей $\varphi: \partial G \rightarrow \mathbb{R}^{N}$. Обозначим через $[G, \varphi]_{\Gamma}$ множество всех линейных сетей из $[G, \varphi]$, параллельных $\Gamma$. Общая задача, которая будет решена в настоящем параграфе (см. теорему 4.1), состоит в следуюшем.

ЗАДАЧА 4.1. Описать структуру множества $[G, \varphi]_{\Gamma}$.

В следующем параграфе в качестве следствий теоремы 4.1 мы получим описание всех взвешенных локально минимальных сетей данного типа с данной гранищей, (см. теорему 5.1).

Выше мы определили разбиение произвольного графа, а значит, и произвольной сети на невырожденные компоненты. При рассмотрении классов параллельности линейных сетей эти компоненты, как легко видеть, независимы в следующем смысле.

ПРЕДЛОЖЕНИЕ 4.1. Пусть $\Gamma_{1}, \ldots, \Gamma_{k}$ - невырожденные компоненты линейной погруженной сети $\Gamma$ с границей $\varphi$ и $\varphi_{i}-$ граница сети $\Gamma_{i}$. Тогда

$$
[\Gamma, \varphi]_{\Gamma}=\left[G_{1}, \varphi_{1}\right]_{\Gamma_{1}} \times \ldots \times\left[G_{k}, \varphi_{k}\right]_{\Gamma_{k}}
$$

Отметим, что предложение 4.1 позволяет свести задачу описания множества $[G, \varphi]_{\Gamma}$ к задаче описания таких множеств для невырожденных компонент.

Пусть $\Gamma$, как и выше, - погруженная линейная сеть типа $G$ с границей $\varphi$. Для описания множества $[G, \varphi]_{\Gamma}$ нам понадобится следующая линейная система уравнений. Фиксируем в пространстве $\mathbb{R}^{N}$ некоторые евклидовы координаты. Фиксируем некоторую ориентацию сети $Г$ и будем называть эту ориентацию исходной. Если $e$-произвольное ребро сети $\Gamma$, ориентированное одним из двух возможных способов, то положим $\varepsilon(e)$ равным 1 , если эта ориентация ребра $e$ совпадает с исходной. В противном случае положим $\varepsilon(e)=-1$. Пусть $\nu(e)-$ координаты единичного вектора направления ребра $e$ сети $\Gamma$ в исходной ориентации.

Разобьем граф $G$ на невырожденные компоненты $G_{i}$, и пусть $\Gamma_{i}=\left.\Gamma\right|_{G_{i}}-$ соответствуюшие сети. Рассмотрим произвольную компоненту $\Gamma_{i}$. Обозначим через $M_{1}, \ldots, M_{n}$ координаты образов граничных вершин $v_{1}, \ldots, v_{n}$ сети $\Gamma_{i}$. Пусть $e_{j}-$ единственное граничное ребро, инцидентное вершине $v_{j}$. Для каждого ребра $e_{j}$, отличного от $e_{1}$, фиксируем некоторый ориентированньй путь $\gamma_{j}$ в $\Gamma_{i}$, соединяющий ребра $e_{1}$ и $e_{j}$, начинаюшийся в $v_{1}$ и заканчиваюшийся в $v_{j}$. Отметим, что ориентация пути $\gamma_{j}$ определяет значения чисел $\varepsilon(e)$ для всех ребер $e$ из $\gamma_{j}$. Далее, пусть $c_{1}, \ldots, c_{k}$ - циклы из $\Gamma_{i}$, соответствуюшие некоторой фундаментальной системе циклов графа $G_{i}$, на каждом из которых фиксирована ориентация. Отметим, что объединение построенных систем фундаментальных циклов по всем невырожденным компонентам $G_{i}$ образует фундаментальную систему циклов подвижного подграфа $\widehat{G}$ графа $G$. Как и в случае путей $\gamma_{j}$, ориентация каждого цикла $c_{q}$ определяет значения чисел $\varepsilon(e)$ для всех ребер $e$ из $c_{q}$.

Каждому ребру $e$ сети $\Gamma_{i}$ поставим в соответствие вещественную переменную $\lambda_{e}$. Рассмотрим следуюшую линейную систему уравнений на вещественные 
переменные $\lambda_{e}$, записанную в векторной форме:

$$
\begin{array}{ll}
\sum_{e: e \in \gamma_{j}} \varepsilon(e) \nu(e) \lambda_{e}=M_{j}-M_{1} & \forall \gamma_{j}, \\
\sum_{e: e \in c_{q}} \varepsilon(e) \nu(e) \lambda_{e}=0 & \forall c_{q} .
\end{array}
$$

Эта система линейных уравнений называется фундаментальной системой невырожденной компоненты $\Gamma_{i}$, а ее ранг - рангом $\mathrm{rk} \Gamma_{i}$ компоненты $\Gamma_{i}$. Отметим, что уравнения фундаментальной системы, соответствуюшие путям $\gamma_{j}$, выражают векторы $M_{1} M_{j}$ в виде суммы векторов-звеньев ориентированной ломаной $\gamma_{j}$. Уравнения фундаментальной системы, отвечаюшие циклам $c_{q}$, означают замкнутость каждой ориентированной ломаной $c_{q}$ : сумма всех векторов-звеньев такой ломаной равна нулю.

ОПРЕДЕЛЕНИЕ. Фундаментальной системой сети Г называется линейная система уравнений, составленная из фундаментальных систем всех невырожденных компонент сети Г. Рангом $\operatorname{rk}(\Gamma)$ сети Г называется ранг ее фундаментальной системы.

ЗАмечАниЕ. Так как каждое ребро сети Г принадлежит ровно одной ее невырожденной компоненте, фундаментальная система сети Г имеет блочньй вид, поэтому ранг сети Г равен сумме рангов ее невырожденных компонент:

$$
\operatorname{rk}(\Gamma)=\sum_{i} \operatorname{rk}\left(\Gamma_{i}\right)
$$

Отметим, что определенная нами фундаментальная система сети Г зависит от выбора путей $\gamma_{j}$ и циклов $c_{q}$. Тем не менее, имеет место следующий гомологический результат.

ПРЕДЛОЖЕНИЕ 4.2. Все фундаментальные системы сети Г әквивалентны как линейные системы уравнений. В частности, множества решений всех фундаментальных систем совпадают и ранг сети Г определен корректно, т.е. не зависит от выбора фундаментальной системы сети.

ДокАЗАТЕЛьство. Нам понадобится следующая лемма. Пусть $G$-произвольный граф. Ориентируем каждое ребро графа $G$. Обозначим через $C_{i}(G)$ группу клеточных $i$-мерных цепей комплекса $G$ над $\mathbb{Z}$, т.е. множество всех формальных конечных линейных комбинаций $i$-мерных клеток комплекса $G$ с коэффициентами в $\mathbb{Z}$, наделенное естественной операцией сложения, а через $\partial_{i}: C_{i}(G) \rightarrow C_{i-1}(G)-$ граничный гомоморфизм. Тогда группа $H_{1}(G, \mathbb{Z})$ первых гомологий полученного цепного комплекса содержит все ориентированные циклы графа $G$, каждый из которых представляет собой формальную линейную комбинацию ребер графа $G$ с коэффициентами \pm 1 . При этом каждая фундаментальная система циклов графа $G$ образует базис в $H_{1}(G, \mathbb{Z})$. Поэтому имеет место следующий результат.

ЛЕмма 4.1. Каждый ориентированный цикл графа $G$ представим в виде целочисленной линейной комбинации его ориентированных фундаментальных ииклов.

Из леммы 4.1 немедленно вытекают следующие результаты. 
ЛЕмма 4.2. Пусть Г - произвольная погруженная линейная сеть, $\left\{\lambda_{e}\right\}-$ некоторое решение ее фундаментальной системы и с- произвольный ориентированный иикл сети Г, все ребра которого - внутренние для Г. Тогда

$$
\sum_{e: e \in c} \varepsilon(e) \nu(e) \lambda_{e}=0
$$

Поэтому фундаментальная система, полученная из данной фундаментальной системы заменой базиса фундаментальных циклов подвижсного подграфа, әквивалентна исходной системе.

ЛЕмма 4.3. Пусть $\delta$ - ориентированный путь в невырожденной компоненте сети $Г$, начинающийся в е $e_{1}$ и заканчивающийся в $e_{j}$. Тогда фундаментальная система уравнений сети Г, полученная из исходной фундаментальной системы сети Г заменой уравнений для $\gamma_{j}$ на уравнения

$$
\sum_{e: e \in \delta} \varepsilon(e) \nu(e) \lambda_{e}=M_{j}-M_{1}
$$

для $\delta$, әквивалентна исходной фундаментальной системе.

Утверждение предложения вытекает из лемм 4.2 и 4.3. Доказательство закончено.

Изучим, как связаны решения фундаментальной системы сети $Г$ и элементы множества $[G, \varphi]_{\Gamma}$. Если $\Gamma^{\prime}$ - произвольная параллельная $\Gamma$ линейная сеть типа $G$ с гранищей $\varphi$, то набор длин ребер сети $\Gamma^{\prime}$ удовлетворяет фундаментальной системе сети $Г$.

Обратно, пусть $\left\{\lambda_{e}\right\}$ - некоторое решение фундаментальной системы. Покажем, что если все $\lambda_{e}>0$, то сушествует параллельная $\Gamma$ линейная сеть типа $G$ с гранищей $\varphi$, длины ребер которой равны соответствующим значениям $\lambda_{e}$. Для этого сначала для произвольного решения $\left\{\lambda_{e}\right\}$ фундаментальной системы построим линейную сеть $\Gamma^{\prime}$ из $[G, \varphi]$. Легко видеть, что если мы реализуем все невырожденные компоненты сети $\Gamma^{\prime}$ на соответствуюших им подмножествах множества $M$, то мы, тем самым, реализуем и всю сеть $\Gamma^{\prime}$. Поэтому в силу блочного вида фундаментальной системы без ограничения общности можно предполагать, что граф $G$ совпадает со своей единственной невырожденной компонентой.

Пусть $T$ - остовное дерево графа $G$ с границей $\partial T=\partial G$. Выберем все пути $\gamma_{j}$ лежашими в $T$. Напомним, что у графа $G$, а значит, и у дерева $T$ фиксирована исходная ориентация. Построим линейное дерево $\Upsilon$ такое, что $\Upsilon\left(v_{1}\right)=M_{1}$, и вектор $\Upsilon(e)$, соответствующий произвольному ориентированному ребру $e$ из $T$, равен $\lambda_{e} \nu(e)$. Так как набор чисел $\left\{\lambda_{e}\right\}$ удовлетворяет фундаментальной системе, то гранища $\partial \Upsilon$ дерева $\Upsilon$ совпадает с $\partial \Gamma$ (отметим, что по определению параметризуюшее дерево $T$ сети $\Upsilon$ является остовным подграфом в $G$ ).

Так как отображение $\Upsilon$ определено на всех вершинах графа $G$, то оно по линейности продолжается до единственной линейной сети $\Gamma^{\prime}: G \rightarrow \mathbb{R}^{N}$. Покажем, что вектор $\Gamma^{\prime}(e)$, соответствуюший произвольному ориентированному ребру $e$ из $G$, равен $\lambda_{e} \nu(e)$. 
Если $e \in T$, то доказывать нечего. Далее, пусть $e \notin T$ и $c$ - единственный цикл в графе $T \cup\{e\}$, ориентированный произвольным образом. Обозначим через $v$ и $v^{\prime}$ инцидентные $e$ вершины из $G$. В силу леммы 4.2 имеем

$$
\varepsilon(e) \nu(e) \lambda_{e}=-\sum_{e^{\prime}: e^{\prime} \in c \cap T} \varepsilon\left(e^{\prime}\right) \nu\left(e^{\prime}\right) \lambda_{e^{\prime}}
$$

Осталось заметить, что в правой части стоит разность радиус-векторов точек $\Upsilon(v)$ и $\Upsilon\left(v^{\prime}\right)$, что и требовалось доказать.

Обозначим через $\Lambda$ аффинное подпространство в $\mathbb{R}^{m}$, являющееся множеством решений фундаментальной системы сети $\Gamma$. Пусть $\psi: \Lambda \rightarrow[G, \varphi]-$ построенное только что отображение, ставящее в соответствие каждому набору $\left(\ldots, \lambda_{e}, \ldots\right) \in \Lambda$ соответствующую линейную сеть из $[G, \varphi]$, точнее, вектор координат подвижных вершин этой сети. Очевидно, что $\psi$ - аффинное отображение.

Легко видеть, что имеет место следуюшее утверждение.

ПрЕДЛОЖЕНИЕ 4.3. Сеть $\Gamma^{\prime} \in[G, \varphi], \quad \Gamma^{\prime}=\psi\left(\ldots, \lambda_{e}, \ldots\right)$, параллельна сети Г, если и только если все $\lambda_{e}$ строго положительны. Иными словами,

$$
[G, \varphi]_{\Gamma}=\psi\left(\Lambda \cap \operatorname{int} \mathbb{R}_{+}^{m}\right),
$$

где через $\mathbb{R}_{+}^{m}$ обозначена внутренность положительного ортанта в пространстве $\mathbb{R}^{m}$.

Предложение 4.3 позволяет легко доказать следуюший результат.

Лемма 4.4. Отображсние $\psi$ взаимно однозначно с образом, поэтому $\psi-$ аффинное вложение.

ДокАЗАТЕЛЬСТво. Так как положительный ортант - открытое множество, взаимная однозначность отображения $\psi$ вытекает из аффинности этого отображения и того факта, что расположение вершин линейной сети однозначно определяет вектор длин ее ребер.

Теперь все готово, чтобы доказать следующую теорему.

TЕОРема 4.1. Пусть $\Gamma$ - погруженная линейная сеть типа $G$ с границей $\varphi$ в $\mathbb{R}^{N}$. Обозначим через $m$ число ребер графа $G$ и через $r-$ ранг $\operatorname{rk}(\Gamma)$ сети $\Gamma$. Тогда $[G, \varphi]_{\Gamma}$ - открытое выпуклое многогранное подмножество в $\psi(\Lambda)($ вообще говоря, не ограниченное), его размерность равна $m-r$ и количество граней максимальной размерности не превосходит $\mathrm{m}$.

ДокАЗАТЕЛЬСТВо. В силу предложения 4.3

$$
[G, \varphi]_{\Gamma}=\psi\left(\Lambda \cap \operatorname{int} \mathbb{R}_{+}^{m}\right) .
$$

Теперь выпуклость, открытость и многогранность множества $[G, \varphi]_{\Gamma}$ в аффинном подпространстве $\psi(\Lambda) \subset[G, \varphi]$ вытекает из аффинности отображения $\psi$ и выпуклости, открытости и многогранности в $\Lambda$ множества $\Lambda \cap \operatorname{int} \mathbb{R}_{+}^{m}$. В частности, размерность множества $[G, \varphi]_{\Gamma}$ равна размерности пространства $\Lambda$, т.е. равна $m-r$. Для завершения доказательства достаточно воспользоваться следующей очевидной леммой. 
Лемма 4.5. Пусть $X$ - внутренняя точка положительного ортанта $\mathbb{R}_{+}^{m}$ и П - проходящее через $X$ аффинное подпространство в $\mathbb{R}^{m}$, пересекающее $\mathbb{R}_{+}^{m}$ по множеству $W$. Тогда $W$ - выпуклое многогранное множество, количество граней максимальной размерности которого не превосходит $m$.

Теорема доказана.

Оказывается, еслигранища сети Г удовлетворяет некоторым естественным ограничениям, то теорему 4.1 можно усилить. Пусть, как и выше, $\Gamma: G \rightarrow \mathbb{R}^{N}$ - погруженная линейная сеть. Определим геометрическую гранииу $\partial_{g} \Gamma$ сети Г следуюшим образом. Вершину $v$ сети $Г$ отнесем к $\partial_{g} \Gamma$, если и только если сушествует такая проходящая через $v$ гиперплоскость в $\mathbb{R}^{N}$, что одно из ограниченных ею открытых полупространств содержит внутренности всех ребер из $\Gamma$, инщидентных $v$.

Имеет место следуюший результат.

ПРЕДЛОЖЕНИЕ 4.4. Погруженная линейная сеть содержится в выпуклой оболочке своей геометрической границы.

ДоКАЗАТЕльство. Предположим противное. Тогда выпуклая оболочка всей сети содержит выпуклую оболочку ее геометрической гранищы как собственное подмножество. Отсюда немедленно вытекает, что существует вершина $v$ сети, не принадлежащая геометрической гранище, лежащая на гранище выпуклой оболочки сети и такая, что одна из опорных гиперплоскостей к выпуклой оболочке сети пересекает эту оболочку в точности по вершине $v$. Но последнее означает, что $v$ принадлежит геометрической границе сети, что невозможно. Доказательство закончено.

Из предложения 4.4 и теоремы 4.1 вытекает следующий результат.

СледСТВИЕ 4.1. В предположениях теоремы 4.1 если граница ч сети Г содержит геометрическую граничу $\partial_{g} \Gamma$ әтой сети, то множество $[G, \varphi]_{\Gamma}$ является внутренностью выпуклого (ограниченного) многогранника размерности $m-r$, количество граней максимальной размерности которого не превосходит $m$.

ЗАмЕЧАНИЕ. Легко построить пример погруженной линейной сети Г, граница $\varphi$ которой не содержит геометрической границы $\partial_{g} \Gamma$, однако множество $[G, \varphi]_{\Gamma}$ ограничено. Действительно, пусть граф $G$ - цикл из трех ребер, граница $\partial G$ которого состоит из каких-нибудь двух его вершин. Зададим линейную сеть $\Gamma: G \rightarrow \mathbb{R}^{2}$ как отображение цикла $G$ на некоторьй треугольник $A B C$ в плоскости так, что граница $\partial \Gamma$ сети $\Gamma$ переводит $\partial G$ в пару вершин этого треугольника. Ясно, что пространство $[G, \varphi]_{\Gamma}$ в этом случае состоит из одной точки, хотя граница $\varphi$ и не содержит геометрической границы сети Г. Было бы интересно найти критерий ограниченности множества $[G, \varphi]_{\Gamma}$ в терминах геометрии гранищы $\varphi$.

Пусть $\Gamma: G \rightarrow \mathbb{R}^{N}$ - погруженная линейная сеть с гранищей $\varphi$. Для каждой вершины $v$ графа $G$ назовем локусом вершины $v$ замыкание подмножества пространства $\mathbb{R}^{N}$, состоящего из точек вида $\Gamma^{\prime}(v)$ по всем линейным сетям $\Gamma^{\prime}$ из пространства $[G, \varphi]_{\Gamma}$. Локус вершины $v$ для $\varphi$ граффа $G$ обозначим через $\operatorname{loc}_{\Gamma}(v)$. Имеет место следующее очевидное следствие из теоремы 4.1 . 
СлЕДСТвИЕ 4.2. В сделанных выше обозначениях локус $\operatorname{loc}_{\Gamma}(v)$ произвольной подвижной вершины $v$ графа $G$ представляет собой выпуклое многогранное подмножество в $\mathbb{R}^{N}$, являющееся проекцией многогранного множества $[G, \varphi]_{\Gamma}$ на соответствующее этой вершине $N$-мерное координатное подпространство в конфигурационном пространстве подвижных вершин графа $G$.

\section{$\S 5$. Взвешенные минимальные сети в $\mathbb{R}^{N}$}

В данном параграфе мы используем полученные выше общие результаты для описания структуры множества лежащих в $\mathbb{R}^{N}$ взвешенных минимальных сетей фиксированного типа с данной гранищей.

5.1. Основные определения и факты. Начнем с определения взвешенных минимальных сетей в римановом многообразии $W$. Напомним, что ребра погруженной сети $Г$ являются регулярными кривыми, и поэтому для каждого ребра $e$ сети Г определена длина $\ell(e)$.

Пусть $G$ - взвешенный граф, т.е. $G$ - некоторый топологический граф, на множестве ребер которого задана положительная вещественная функция $\omega$, называемая весовой функиией. Каждую сеть вида $\Gamma: G \rightarrow W$ назовем в этом случае взвешенной сетью типа $G$. Из определения понятия ребра сети вытекает, что функцию $\omega$ можно рассматривать как функцию на множестве всех ребер сети $\Gamma$, что мы и будем делать. Взвешенной длиной $\ell_{\omega}(\Gamma)$ взвешенной сети $\Gamma$ типа $G$ назьвается следующая величина:

$$
\ell_{\omega}(\Gamma)=\sum_{e \in E(\Gamma)} \omega(e) \ell(e)
$$

Пусть опять $\Gamma: G \rightarrow W$ - произвольная сеть, $P$ - любая точка графа $G$ и $G_{U}-$ локальный граф с центром в $P$. Сеть с границей, равная ограничению отображения $\Gamma$ на $G_{U}$, называется локальной сетью с чентром в точке $P$ и обозначается через $\Gamma_{U}$. При этом ограничение отображения $\Gamma$ на каноническую границу $\partial G_{U}$ локального графа $G_{U}$ называется канонической граничей локальной сети $\Gamma_{U}$ и обозначается через $\partial \Gamma_{U}$. Отметим, что если $G$ - взвешенный граф и $G_{U}-$ некоторый его локальньй граф, то как на $G_{U}$, так и на соответствующей локальной сети $\Gamma_{U}$ естественным образом индуцируется весовая функция. Поэтому для каждой локальной сети взвешенной сети Г определена взвешенная длина.

Взвешенная сеть $\Gamma$ типа $G$, затягиваюшая конечное множество $M \subset W$ точек риманова многообразия $W$, называется взвешенной минимальной сетью с границей $M$ или по отношению $\kappa$ границе $M$, если для любой ее точки $P$ некоторая локальная сеть $\Gamma_{U}$ с центром в $P$ имеет наименьшую взвешенную длину среди всех сетей типа $G_{U}$ с той же границей. Иногда, там где это не вызовет недоразумений, мы будем называть взвешенные минимальные сети просто минимальными сетями, опуская слово "взвешенные".

Следующая теорема полностью описывает локальное устройство взвешенных минимальных сетей в произвольном римановом многообразии (см. [42], [36]).

ПРЕДЛОЖЕНИЕ 5.1 (о локальной структуре). Взвешенная сеть Г с границей $M$ является минимальной, если и только если имеют место следующие свойства: 
1) все ребра сети - отрезки геодезических;

2) для каждой подвижной вершинь $v$ линейная комбинация единичных векторов направлений входящих в $v$ ребер с коэффичиентами - весами этих ребер, - равна нулю.

ЗАмечАниЕ. Вообще говоря, взвешенная минимальная сеть $\Gamma: G \rightarrow W$ может содержать кратные ребра. Из предложения 5.1 вытекает, что если каждые две точки многообразия $W$ соединяет не более одной геодезической (например, $W$ область в $\mathbb{R}^{N}$ или в пространстве Лобачевского), то образы всех кратных ребер совпадают. Более того, если в этом случае перестроить взвешенный граф $G$, заменив каждый набор $e_{1}, \ldots, e_{p}$ кратных ребер на одно из этих ребер с весом, равным $\sum_{i=1}^{p} \omega\left(e_{i}\right)$, и определить на полученном графе $\bar{G}$ сеть $\bar{\Gamma}$, совпадающую с $\Gamma$, то взвешенная сеть $\bar{\Gamma}$ вновь будет минимальной с той же границей, что и сеть Г. Описанную операцию на взвешенных графах и соответствуюших сетях назовем склейкой кратных ребер, а полученные в результате склейки кратных ребер граф $\bar{G}$ и сеть $\bar{\Gamma}$ будем обозначать через $g(G)$ и $g(\Gamma)$ соответственно.

СлЕДСТВИЕ 5.1. Пусть Г является взвешенной минимальной сетью и $v-$ произвольная ее подвижная вершина. Тогда степень вериинь $v$ не меньше 2 и веса ребер, иниидентных $v$, удовлетворяют “правилу многоугольника": вес каждого из этих ребер не больше суммы весов всех остальных этих ребер. В частности, если направления всех ребер, инцидентных вериине $v$, коллинеарны, то эти ребра разбиваются на два класса с одинаковыми суммарными весами. Поэтому веса ребер, инцидентных подвижной вериине степени 2, равны межсду собой.

ЗАмечАниЕ. Пусть $G$ - некоторый взвешенный граф и $\Gamma: G \rightarrow W-$ взвешенная минимальная сеть. Если некоторая вершина степени 2 сети $\Gamma$ - подвижная, то иншидентные этой вершине ребра по следствию 5.1 необходимо имеют одинаковые веса и поэтому стыкуются под углом в $180^{\circ}$. Отсюда вытекает, что если выбросить из множества вершин параметризуюшего графа $G$ подвижные вершины степени 2 , иншидентные разным ребрам одного веса ${ }^{1}$, и “укрупнить" при этом ребра, склеивая каждую пару ребер из $G$, инщидентных такой вершине, в одно ребро того же веса, то получим, очевидно, новую взвешенную минимальную сеть, образ которой совпадает с образом сети $Г$.

Обратно, из любой взвешенной сети $\Gamma$, не меняя ее образ, можно получить другую взвешенную сеть, выбрав на ребрах параметризуюшего графа $G$ произвольные внутренние точки и добавив их к множеству подвижных вершин. При этом надо сделать очевидную перестройку ребер и весовой функции сети. Более того, по предложению 5.1 при любой такой перестройке сети каждая взвешенная минимальная сеть остается взвешенной минимальной сетью.

ОПРЕДЕЛЕНИЕ. Каждую не инцидентную петле подвижную вершину степени 2 произвольного взвешенного граф̆а $G$, а также соответствующую вершину произвольной сети $\Gamma$ типа $G$ будем называть фиктивной.

\footnotetext{
${ }^{1}$ Отметим, что если граф $G$ содержит вершину степени 2 , инцидентную одному ребру, то это ребро является петлей и графф $G$ совпадает с этой петлей.
} 
Для сетей в многообразиях, каждые две точки которых соединяются единственной геодезической, понятие фиктивной вершины можно естественно расширить. Пусть теперь $G$ - произвольньй взвешенньй граф (возможно, с кратными ребрами), параметризуюший сеть $\Gamma$ в многообразии $W$, обладающем указанным свойством, и $g(G)$ - результат склейки кратных ребер графа $G$.

ОПРЕДЕЛЕНИЕ. Вершину $v$ сети $Г$ типа $G$ (соответственно графа $G$ ) назовем фиктивной, если и только если фиктивной является соответствуюшая вершина графа $g(G)$.

Другими словами, вершина $v$ сети Г является фиктивной, если она подвижна, смежна ровно с двумя вершинами, скажем $v_{1}$ и $v_{2}$, и сумма весов ребер, соединяющих $v$ с $v_{1}$, равна сумме весов ребер, соединяющих $v$ с $v_{2}$. Отметим, что на случай таких сетей естественно переносится операция удаления фиктивных вершин.

Имеет место следующее утверждение.

УТВЕРЖДЕНИЕ 5.1. Пусть каждье две точки риманова многообразия $W$ соединяет единственная геодезическая. Тогда взвешенная сеть Г с границей $\partial Г$ является минимальной, если и только если минимальной является взвешенная сеть, полученная из Г удалением всех фиктивных вершин.

ЗАмечАниЕ. Пусть $\Gamma$ - произвольная взвешенная минимальная сеть с границей $\partial Г$. Если для некоторой граничной вершины выполняется условие предложения 5.1, характеризуюшее подвижные вершины, т.е. линейная комбинация векторов направлений ребер, входящих в эту вершину, с коэффициентами - весами этих ребер, - равна нулю, то такую вершину можно исключить из границы, объявив ее подвижной, что не изменит свойства сети Г быть взвешенной минимальной. Такие граничные вершины будем называть ложными. Легко видеть, что взвешенные минимальные сети, затягиваюшие границы обшего положения, не содержат ложных вершин.

ЗАмечАниЕ. Пусть $\Gamma$ - произвольная взвешенная минимальная сеть с границей $\partial Г$. В соответствии с предложением 5.1 каждая невырожденная компонента сети $Г$ сама является взвешенной минимальной сетью.

Множество всех вершин степени 1 произвольного граффа (сети) назовем әффективной границей этого графа (сети). Итак, граница каждой невырожденной компоненты графа (сети) эффективна.

Задача описания взвешенных минимальных сетей общего вида сводится к задаче описания невырожденных взвешенных минимальных сетей. А именно, имеет место следуюший очевидный результат.

УТВЕРЖДЕНИЕ 5.2. Взвешенная сеть $G$ с границей ӘГ является минимальной тогда и только тогда, когда каждая ее невырожденная компонента взвешенная минимальная сеть по отношению к своей границе.

Понятие взвешенной длины естественным образом распространяется на произвольные взвешенные геодезические сети (не обязательно погруженные). Пусть $G-$ взвешенный граф с весовой функцией $\omega$ и $\Gamma$ - произвольная геодезическая сеть типа $G$. Обозначим через $\ell_{\omega}(\Gamma)$ линейную комбинацию длин всех ребер сети $\Gamma$ с коэффициентами - весами соответствуюших ребер из $G$ и назовем это число взвешенной длиной геодезической сети $Г$. Функция $\ell_{\omega}$ определена на пространстве 
всех геодезических сетей топологии $G$. Отметим, что для геодезических сетей $\Gamma$ без вырожденных ребер, т.е. для погруженных геодезических сетей, определенная только что функция совпадает со взвешенной длиной, определенной выше для погруженных сетей.

Начиная с этого места, мы снова будем предполагать, что $W=\mathbb{R}^{N}$. Напомним, что через $\mathfrak{L}(G)$ мы обозначаем пространство всех линейных сетей топологии $G$. На этом пространстве, как было только что объяснено, определена функция $\ell_{\omega}$ взвешенной длины. Следующее предложение доказьвается точно так же, как и соответствуюшее предложение из [47].

ПРЕДЛОЖЕНИЕ 5.2. Функиия $\ell_{\omega}: \mathfrak{L}(G) \rightarrow \mathbb{R}$ выпукла вниз.

СлЕДСТВИЕ 5.2. Оораничение функиии $\ell_{\omega}$ на $[G, \varphi]$ выпукло.

Следующее предложение описывает множество экстремумов функции $\ell_{\omega}$ : $[G, \varphi] \rightarrow \mathbb{R}$ (его доказательство также может быть легко получено из соответствующего результата в [47]).

ПРЕДЛОЖЕНИЕ 5.3. Множество әкстремумов функиии $\ell_{\omega}:[G, \varphi] \rightarrow \mathbb{R}$ непусто, выпукло (а значит, линейно связно), ограничено и состоит из точек абсолютного минимума.

Множество всех минимумов функции $\ell_{\omega}:[G, \varphi] \rightarrow \mathbb{R}^{N}$ обозначим через $[G, \varphi]_{\min }$. Получим теперь необходимое и достаточное условия того, что точка $x \in[G, \varphi]$ является точкой минимума. Для этого напишем формулу первой вариации функции $\ell_{\omega}$.

Пусть $G$ - взвешенный граф, $\omega$ - весовая функция, $\Gamma$ - произвольная линейная сеть из $\mathfrak{L}(G)$ и $v$ - произвольная ее вершина. Обозначим через $N_{v}$ линейную комбинацию единичных векторов направлений входящих в вершину $v$ невырожденных ребер с коэффишиентами - весами этих ребер. Далее, пусть $D(v)$ - множество всех вершин, смежных с $v$, для которых ребра, соединяющие их с вершиной $v$, вырождены.

Рассмотрим произвольную деформацию $\Gamma_{t}$ сети $\Gamma$ в классе $\mathfrak{L}(G)$, и пусть $E_{v}-$ начальный вектор скорости движения вершины $v$ при этой деформации.

ПРЕДЛОЖЕНИЕ 5.4 (первая вариация взвешенной длины). Имеет место следующее соотношение:

$$
\left.\frac{d \ell_{\omega}\left(\Gamma_{t}\right)}{d t}\right|_{t=0}=\sum_{v}\left(\left\langle N_{v}, E_{v}\right\rangle+\frac{1}{2} \sum_{v^{\prime} \in D(v)} \omega\left(v v^{\prime}\right)\left|E_{v}-E_{v^{\prime}}\right|\right)
$$

где $\omega\left(v v^{\prime}\right)-$ вес ребра сети $\Gamma$, соединяющего $v$ и $v^{\prime}$.

Таким образом, сеть $Г$ является точкой абсолютного минимума функции $\ell_{\omega}$ : $[G, \varphi] \rightarrow \mathbb{R}$, если и только если для любых семейств векторов $E_{v}$, равных нулю в неподвижных вершинах, выражение

$$
\sum_{v}\left(\left\langle N_{v}, E_{v}\right\rangle+\frac{1}{2} \sum_{v^{\prime} \in D(v)} \omega\left(v v^{\prime}\right)\left|E_{v}-E_{v^{\prime}}\right|\right)
$$

неотрицательно. 
СлЕДСТВИЕ 5.3. Пусть $G$ - взвешенный граф с весовой функиией $\omega$, а $\Gamma$ линейная сеть из $[G, \varphi]$, не содержащая вырожденных ребер. Тогда Г является точкой абсолютного минимума функиии взвешенной длины $\ell_{\omega}$, m.е. $\Gamma \in[G, \varphi]_{\min }$, если и только если $\Gamma$ - взвешенная минимальная сеть c границей $\varphi$. В частности, если все абсолютные минимумы функчии взвешенной длины на пространстве $[G, \varphi]$ содержат вырожденные ребра, то не существует взвешенной минимальной сети типа $G$ с границей $\varphi$.

Перейдем теперь к формуле второй вариации длины. Нам не понадобится формула второй вариации в обшем случае, поэтому мы выпишем ее лишь в предположении, что все вершины деформируемой сети равномерно движутся по прямым. Деформашия, удовлетворяюшая указанному свойству, называется линейной.

ПрЕДлОЖЕНиЕ 5.5 (вторая вариация длины отрезка). Пусть $\left[a_{t}, b_{t}\right]-$ nроизвольная линейная деформачия отрезка $\left[a_{0}, b_{0}\right], t \geqslant 0, u\left|a_{0} b_{0}\right| \neq 0$, m.е. отрезок $\left[a_{0}, b_{0}\right]$ невырожден. Тогда

$$
\left.\frac{d^{2}\left|a_{t} b_{t}\right|}{d t^{2}}\right|_{t=0}=\frac{\left|b_{0}-a_{0}\right|^{2}\left|b^{\prime}-a^{\prime}\right|^{2}-\left\langle b_{0}-a_{0}, b^{\prime}-a^{\prime}\right\rangle^{2}}{\left|b_{0}-a_{0}\right|^{3}}
$$

где $a^{\prime}$ и $b^{\prime}$ - скорости кривых $a_{t}$ и $b_{t}$ в начальный момент времени $t=0$.

Доказательство получается прямым подсчетом.

СлеДСтвИЕ 5.4. В предположениях предложения 5.5 вторая производная длинь отрезка неотрицательна и равна нулю, если и только если разность $b^{\prime}-a^{\prime}$ векторов начальных скоростей вариации отрезка коллинеарна направлению отрезка. В частности, вторая производная длины отрезка равна нулю, если и только если все отрезки $\left[a_{t}, b_{t}\right]$ рассматриваемой линейной вариачии взаимно параллельны.

5.2. Пространство взвешенных минимальных сетей данного типа с фиксированной границей. Мы начнем с аналога доказанной Хвангом [15] теоремы единственности плоских локально минимальных деревьев данного типа с данной гранищей. Для этого мы расширим класс фиктивных вершин так. Пусть $\Gamma: G \rightarrow \mathbb{R}^{N}$ - взвешенная минимальная сеть с границей $\varphi$. Подвижная вершина $V$ сети $\Gamma$ называется слабо фиктивной, если все инцидентные ей ребра взаимно параллельны. Из минимальности сети $Г$ вытекает, что ребра сети $\Gamma$, инцидентные слабо фиктивной вершине $V$, а также соответствуюшие ребра графа $G$ разбиваются на два класса с одинаковым суммарным весом. При этом образы любых двух таких ребер пересекаются по точке тогда и только тогда, когда эти ребра принадлежат разным классам. Отметим, что фиктивные вершины являются слабо фиктивными для любой взвешенной минимальной сети $\Gamma$, т.е. это свойство не зависит от выбора отображения Г в классе взвешенных минимальных сетей. В общем случае слабо фиктивные вершины могут перестать быть таковыми при сколь угодно малом изменении отображения Г в классе взвешенных минимальных сетей. Приведем пример.

ПримеР. Пусть $G$ - граф, состояший из четырех ребер, стыкуюшихся в одной обшей вершине $v$, и весовая функция постоянна и равна 1 . Пусть $\partial G-$ эффективная 
граница графа $G$, которая состоит из четырех вершин. Рассмотрим граничное отображение $\varphi$, переводящее множество $\partial G$ в четыре различных точки $M_{1}, \ldots, M_{4}$, лежащие на одной прямой $l$. Будем предполагать без ограничения общности, что точки $M_{i}$ занумерованы последовательно. Тогда для любого расположения образа $A$ вершины $v$ на интервале $\left(M_{2}, M_{3}\right)$ соответствуюшая линейная сеть $\Gamma$ является взвешенной минимальной сетью типа $G$ с гранищей $\varphi$. Вершина $\Gamma: v \mapsto A$ этой сети слабо фиктивна. Отметим, что в этом случае имеется однопараметрическое семейство взвешенных минимальных сетей типа $G$ с границей $\varphi$.

Если же сколь угодно мало изменить отображение $\varphi$ так, чтобы точки $M_{i}$ стали вершинами плоского выпуклого четырехугольника, то для такого граничного отображения существует единственная взвешенная минимальная сеть $\Gamma$ типа $G$. Образ $A$ вершины $v$ сети $\Gamma$ совпадает с точкой пересечения диагоналей граничного четырехугольника. Ясно, что вершина $\Gamma: v \mapsto A$ в этом случае не является слабо фиктивной.

ПрЕДЛОЖЕНИЕ 5.6. Взвешенное минимальное дерево $Г: G \rightarrow \mathbb{R}^{N}$ является единственной взвешенной минимальной сетью типа $G$ с границей только если Г не содержит слабо фиктивных вериин.

ДокАЗАТЕльство. Если сеть Г содержит слабо фиктивную вершину, то, очевидно, эту вершину можно смещать вдоль прямой, содержащей инцидентные этой вершине ребра. Поэтому в этом случае сеть не единственна.

Докажем теперь обратное утверждение, т.е. что из отсутствия фиктивных вершин вытекает единственность сети Г. Предположим противное, т.е. сушествует отличная от Г взвешенная минимальная сеть $\Gamma^{\prime}: G \rightarrow \mathbb{R}^{N}$ с границей $\partial \Gamma^{\prime}=\partial \Gamma$. В силу следствия 5.3 обе сети $Г$ и $\Gamma^{\prime}$ лежат в $[G, \varphi]_{\min }$. Рассмотрим семейство линейных сетей $\Gamma_{t}=(1-t) \Gamma+t \Gamma^{\prime}$, где $t \in[0,1]$. Обозначим через $E_{v}$ вектор скорости деформации $\Gamma_{t}$ в вершине $v$ при $t=0$.

В силу предложения 5.3 все линейные сети $\Gamma_{t}$ лежат в $[G, \varphi]_{\min }$ и, значит, имеют одинаковую взвешенную длину $\ell(t)=\ell_{\omega}\left(\Gamma_{t}\right)$. Поэтому вторая производная функции $\ell(t)$ равна нулю. Из следствия 5.4 вытекает, что для любых смежных вершин $v_{1}$ и $v_{2}$ граффа $G$ разность векторов $E_{v_{1}}$ и $E_{v_{2}}$ параллельна ребру $е$ сети $\Gamma$, соединяющему соответствуюшие $v_{i}$ вершины. В частности, если в одной из вершин, скажем в $v_{1}$, вектор $E_{v_{1}}$ равен нулю, то вектор $E_{v_{2}}$ параллелен ребру $e$.

Рассмотрим множество $F$ всех вершин $v$ графа $G$, в которых вектор $E_{v}$ отличен от нуля. Такие вершины будем называть смещаемыми. Рассмотрим подграф в $G$, порожденный множеством $F$, выберем в этом подграффе произвольную связную компоненту (которая, очевидно, является деревом), и пусть $v$ - вершина степени один в этой компоненте. Так как граф $G$ не содержит фиктивных вершин, а $v-$ подвижная вершина, то степень вершины $v$ в графе $G$ больше двух. Поэтому множество всех несмещаемых вершин из $G$, смежных с $v$, состоит не менее чем из двух элементов. Так как сеть Г не содержит слабо фиктивных вершин, среди несмешаемых вершин, смежных с $v$, существует по крайней мере две, скажем $v_{1}$ и $v_{2}$, таких, что образы $e_{i}$ ребер, соединяюших $v$ с $v_{i}$, не лежат на одной прямой. Так как вершины $v_{1}$ и $v_{2}$ несмешаемы, векторы $E_{v_{1}}$ и $E_{v_{2}}$ равны нулю. Поэтому в соответствии с вышесказанным вектор $E_{v}$ должен быть параллелен обоим отрезкам $e_{i}$, что невозможно. Доказательство закончено. 
Если дерево Г содержит слабо фиктивные вершины, то, как видно из приведенного выше примера, никакой единственности нет. Аналогично, если Г не является деревом, то, вообше говоря, также единственности нет. Приведем соответствующий пример.

ПРимеР. Рассмотрим правильный шестиугольник на плоскости, из каждой вершины которого наружу отложен отрезок, составляющий со смежными сторонами шестиугольника углы в $120^{\circ}$. Если в качестве границы полученной линейной сети выбрать все ее вершины степени 1 и выбрать весовую функцию постоянной, то полученная взвешенная минимальная сеть может быть продеформирована в классе взвешенных минимальных сетей того же типа и с той же границей: шестиугольник можно сжимать и растягивать вдоль прямых, проходяших через граничные ребра. Если к гранище добавить еше хотя бы одну вершину степени 3 , то полученная взвешенная минимальная сеть уже не может быть продеформирована. Причину этого легко понять: если разрезать такую сеть по граничной вершине степени 3 , то она превратится во взвешенное минимальное дерево, которое единственно в соответствии с предложением 5.6.

Напомним, что в работе [47] был полностью изучен случай плоских локально минимальных сетей. Такие сети можно рассматривать как взвешенные минимальные сети с постоянной весовой функцией, вершинами степени не больше трех, без слабо фиктивных вершин и со специальными ограничениями на углы между смежными граничными ребрами (эти углы должны быть не меньше $120^{\circ}$ ). Было показано, что размерность пространства деформаций таких сетей равна числу фундаментальных циклов в подвижном подграфе параметризуюшего графа. Оказывается, если степень вершины может быть больше трех или размерность объемлюшего пространства больше двух, то аналогичный результат места не имеет. Приведем соответствующие примеры.

ПримеР. Рассмотрим квадрат на плоскости и продолжим его стороны за вершины. В результате получим линейную сеть с восемью вершинами степени 1 и четырьмя вершинами степени 4. В качестве границы этой сети выберем эффективную границу, а весовую функцию положим тождественно равной 1 . Легко проверяется, что хотя подвижный подграф полученной взвешенной минимальной сети содержит цикл, эта сеть не может быть продеформирована в классе взвешенных минимальных сетей того же типа и с той же гранищей.

ПримеР. Рассмотрим в пространстве $\mathbb{R}^{3}$ плоскость $\mathbb{R}^{2}$, в которой расположим правильный шестиугольник, из каждой вершины которого наружу выпущены отрезки, образующие со смежными сторонами шестиугольника углы по $120^{\circ}$. Проведем прямую $l$, параллельную одной из сторон шестиугольника и пересекающую шестиугольник по внутренним точкам двух его сторон. Эта прямая разбивает нашу сеть на две компоненты. Легко видеть, что одну из этих двух компонент можно так повернуть относительно прямой $l$, что у полученного пространственного 8-угольника все углы будут равны $120^{\circ}$. Если теперь из вершин 8-угольника, лежаших на $l$, выпустить отрезки, образующие со смежными сторонами 8-угольника углы в $120^{\circ}$, то в результате мы получим локально минимальную сеть, имеющую восемь внутренних вершин - вершин 8-угольника, и восемь граничных - концов отрезков, выпушенных из вершин 8-угольника. Несложно проверить, что построенная сеть не деформируется в классе минимальных сетей с той же границей. Таким 
образом, мы построили пример графа $G$ с постоянной весовой функцией и граничного отображения $\varphi$, для которых существует единственная взвешенная минимальная сеть типа $G$ с границей $\varphi$, несмотря на то, что цикломатическое число подвижного подграфа граффа $G$ равно 1. Идея построения этого примера принадлежит студенту механико-математического факультета МГУ М. В. Пронину.

Следующее предложение позволит нам свести задачу описания множества взвешенных минимальных сетей данного типа с фиксированной границей к теореме 4.1.

ПРЕДЛОЖЕНИЕ 5.7. Пусть $G$ - взвешенный граф с весовой функиией $\omega$, a Г и $\Gamma^{\prime}$ - две взвешенные минимальные сети типа $G$ с одной и той жсе границей $\varphi$. Тогда сети Г и $\Gamma^{\prime}$ параллельны. Поэтому, в частности, если $\Gamma_{t}-$ линейная деформаиия, соединяющая сети $\Gamma$ и $\Gamma^{\prime}$, то все $\Gamma_{t}-$ взаимно параллельнье взвешенные минимальные сети типа $G$ с границей $\varphi$.

ДокАЗАТЕЛЬСтво. Прежде всего покажем, что соответствующие ребра сетей Г и $\Gamma^{\prime}$ параллельны (напомним, что по определению для параллельности сетей этого недостаточно). В силу следствия 5.3 обе сети $\Gamma$ и $\Gamma^{\prime}$ лежат в $[G, \varphi]_{\min }$. Рассмотрим семейство линейных сетей $\Gamma_{t}=(1-t) \Gamma+t \Gamma^{\prime}$, где $t \in[0,1]$. Обозначим через $E_{v}$ вектор скорости деформации $\Gamma_{t}$ в вершине $v$ при $t=0$.

В силу предложения 5.3 все линейные сети $\Gamma_{t}$ лежат в $[G, \varphi]_{\min }$ и, значит, имеют одинаковую взвешенную длину $\ell(t)=\ell_{\omega}\left(\Gamma_{t}\right)$. Поэтому вторая производная функции $\ell(t)$ равна нулю. По следствию 5.4 соответствующие ребра сетей $\Gamma$ и $\Gamma^{\prime}$ параллельны, что и требовалось доказать. Для завершения доказательства предложения достаточно показать, что все линейные сети $\Gamma_{t}$ не содержат вырожденных ребер.

Нам потребуется следующий результат из [47].

УТВЕРЖДЕНИЕ 5.3. Пусть $L-$ прямая в $\mathfrak{L}(G)$, проходящая через погруженную линейную сеть Г. Тогда $L$ содержит не более чем конечное множество линейных сетей, не являющихся погруженными. Поэтому если L - прямая в $[G, \varphi]$, проходящая через погружсенную линейную сеть $\Gamma$, то $L$ также содержит не более чем конечное множество линейных сетей, не являющихся погружсенными.

Вернемся к доказательству предложения. Предположим противное, т.е. среди линейных сетей $\Gamma_{t}$ имеются не погруженные. В силу утверждения 5.3 таких сетей лишь конечное число. Поэтому среди сетей $\Gamma_{t}$ сушествует погруженная сеть $\Gamma^{\prime \prime}$ такая, что между Г и $\Gamma^{\prime \prime}$ есть ровно одна не погруженная сеть $\bar{\Gamma}$ из семейства $\Gamma_{t}$.

Рассмотрим произвольную связную компоненту $\bar{\Gamma}_{d}$ множества всех вырожденных ребер сети $\bar{\Gamma}$. Легко видеть, что эта компонента является подсетью в $\bar{\Gamma}$, содержашей все ребра из $\bar{\Gamma}$, соединяюшие вершины из $\bar{\Gamma}_{d}$. Пусть $\bar{G}_{d}-$ подграф̆ в $G$, параметризуюший $\bar{\Gamma}_{d}$, и $v$ - произвольная вершина из $\bar{G}_{d}$. Обозначим через $e_{1}, \ldots, e_{p}$ все ребра из $\bar{G}_{d}$, инцидентные $v$, а через $f_{1}, \ldots, f_{q}$ - все ребра из $G$, инцидентные $v$ и отличные от всех $e_{i}$. Далее, пусть $\mu_{i}$ и $\nu_{j}$ - единичные векторы направлений, входящих в вершину $\Gamma: v \rightarrow \mathbb{R}^{N}$ ребер $\Gamma: e_{i} \rightarrow \mathbb{R}^{N}$ и $\Gamma: f_{j} \rightarrow \mathbb{R}^{N}$ сети $\Gamma$ соответственно.

Так как сети Г и $\Gamma^{\prime \prime}$ минимальны, имеем

$$
\sum_{j=1}^{q} \omega\left(f_{j}\right) \nu_{j}+\sum_{i=1}^{p} \omega\left(e_{i}\right) \mu_{i}=0, \quad \sum_{j=1}^{q} \omega\left(f_{j}\right) \nu_{j}-\sum_{i=1}^{p} \omega\left(e_{i}\right) \mu_{i}=0
$$


поэтому

$$
\sum_{j=1}^{q} \omega\left(f_{j}\right) \nu_{j}=0, \quad \sum_{i=1}^{p} \omega\left(e_{i}\right) \mu_{i}=0
$$

Пусть $M \subset \mathbb{R}^{N}$ - множество Г-образов вершин из $\bar{G}_{d}$. Так как $\bar{G}_{d}$ содержит по крайней мере одно ребро и сеть $Г$ погруженная, множество $M$ состоит более чем из одной точки. Поэтому выпуклая оболочка $\operatorname{conv}(M)$ множества $M$ является отличным от точки выпуклым многогранником. Пусть $m \in M$ - произвольная вершина выпуклого многогранника conv $(M)$, через которую можно провести гиперплоскость, пересекающуюся с $\operatorname{conv}(M)$ только по $m$. Пусть $v$-вершина из $\bar{G}_{d}$ такая, что $\Gamma(v)=m$. Так как все веса ребер положительны, то для выбранной вершины $v$ равенство

$$
\sum_{i=1}^{p} \omega\left(e_{i}\right) \mu_{i}=0
$$

не выполняется. Противоречие. Тем самым предложение доказано.

СлеДСТВИЕ 5.5. Пусть $G$ - взвешенный граф с весовой функиией $\omega$ и произвольное граничное отображение. Тогда множсество всех минимальных сетей типа $G$ с границей $\varphi$ или пусто, или совпадает с относительной внутренностью выпуклого компакта $[G, \varphi]_{\min }$. Поэтому если существует взвешенная минимальная сеть $\Gamma$ типа $G$ с границей $\varphi$, то

$$
\operatorname{int}[G, \varphi]_{\min }=[G, \varphi]_{\Gamma} \text {. }
$$

ДокАЗАТЕЛЬСтво. Предположим сразу, что существует хотя бы одна взвешенная минимальная сеть $\Gamma$ типа $G$ с границей $\varphi$. Из предложения 5.7 вытекает, что множество всех взвешенных минимальных сетей типа $G$ с данной границей $\varphi-$ выпуклое подмножество в $[G, \varphi]_{\min }$. Покажем, что каждая точка $\Gamma^{\prime}$ из относительной внутренности множества $[G, \varphi]_{\min }$ является взвешенной минимальной сетью.

Выпустим из точки Г луч $r$ в направлении точки $\Gamma^{\prime}$. Так как $\Gamma^{\prime}$ принадлежит относительной внутренности множества $[G, \varphi]_{\min }$, то достаточно близкие к $\Gamma^{\prime}$ точки луча $r$, не лежащие на отрезке $\left[\Gamma, \Gamma^{\prime}\right]$, принадлежат $[G, \varphi]_{\min }$. Пусть $\Gamma^{\prime \prime}-$ любая из таких точек. Из утверждения 5.3 вытекает, что на интервале $\left(\Gamma^{\prime}, \Gamma^{\prime \prime}\right)$ содержится погруженная линейная сеть $\bar{\Gamma}$. По предложению 5.7 отрезок $[\Gamma, \bar{\Gamma}]$, соединяющий две взвешенные минимальные сети одного типа с одной и той же границей, состоит из взвешенных минимальных сетей, что и требовалось доказать.

Таким образом, из теоремы 4.1 и следствия 4.1 вытекает следующий результат.

Теорема 5.1. Пусть Г-взвешенная минимальная сеть типа $G$ в $\mathbb{R}^{N} c$ границей $\varphi$ и весовой функиией $\omega$. Обозначим через $r$ ранг сети $\Gamma$, через $m-$ количество ребер сети $\Gamma$, а через $[G, \varphi]$ - конфигурационное пространство подвижных вериин графа $G$.

Тогда множсество взвешенных минимальных сетей типа $G$ с границей $\varphi$ представляет собой внутренность выпуклого $(m-r)$-мерного многогранника $[G, \varphi]_{\min }$, являющегося множеством всех минимумов функции взвешенной длиньц $\ell_{\omega}:[G, \varphi] \rightarrow \mathbb{R}$. 
Более того, многогранник $[G, \varphi]_{\min }$ имеет не более чем $m$ граней максимальной размерности.

Опишем теперь более подробно, как устроены деформации взвешенных минимальных сетей данной топологии с фиксированной границей. Это описание аналогично соответствующему результату из [47].

Пусть $G$-произвольньй взвешенный граф с весовой функцией $\omega$ и границей $B$ и $\Gamma: G \rightarrow \mathbb{R}^{N}$-взвешенная минимальная сеть с границей $\varphi: B \rightarrow \mathbb{R}^{N}$. Ребро графа $G$ назовем ииклическим, если оно принадлежит хотя бы одному циклу. Обозначим через $\widehat{G}_{B}$ подвижный подграф для $G$. Ребро $е$ из $G$ отнесем к нулевому уровню, если $e$ - циклическое ребро подвижного подграфа $\widehat{G}_{B}$. Ребро, не принадлежащее нулевому уровню, но инцидентное или некоторому ребру нулевого уровня, или слабо фиктивной вершине, назовем ребром первого уровня, а все остальные ребра (т.е. ребра, не лежашие ни на нулевом, ни на первом уровнях) назовем ребрами второго уровня.

Имеет место следующий результат.

ТЕОРема 5.2. Пусть Г- произвольная взвешенная минимальная сеть в $\mathbb{R}^{N}$ типа $G$ с граничей $\varphi$ и $\Gamma_{t}, \quad t \in[0,1]$, - некоторая ее деформация в классе взвешенных минимальных сетей с той же границей $\varphi$. Тогда в процессе деформачии $\Gamma_{t}$ все ребра остаются параллельны себе. При этом ребра второго уровня неподвижны, а для ребер первого уровня остаются неподвижными прямые, содержащие эти ребра.

Для доказательства этой теоремы нам понадобится ряд новых понятий и их свойств.

5.3. Формы Максвелла. Пусть $\Gamma: G \rightarrow \mathbb{R}^{N}$ - произвольная линейная сеть в $\mathbb{R}^{N}, G_{1}, \ldots, G_{k}$ - невырожденные компоненты графа $G$ и $\Gamma_{q}=\left.\Gamma\right|_{G_{q}}-$ соответствуюшие невырожденные компоненты сети $\Gamma$. Рассмотрим некоторую невырожденную компоненту $\Gamma_{q}$. Для каждой граничной вершины $v_{p}$ из $G_{q}$ обозначим через $\nu_{p}$ единичный вектор направления единственного входящего в вершину $\Gamma_{q}: v_{p} \rightarrow \mathbb{R}^{N}$ ребра $\Gamma_{q}: e_{p} \rightarrow \mathbb{R}^{N}$ сети $\Gamma_{q}$, а через $m_{p}$ - радиус-вектор точки $\Gamma_{q}\left(v_{p}\right)$ в $\mathbb{R}^{N}$.

Далее, пусть $\Theta$ - стандартная форма объема в $\mathbb{R}^{N}$. Другими словами, если $\left(V^{1}, \ldots, V^{N}\right)$ - произвольные векторы в $\mathbb{R}^{N}$, то

$$
\Theta\left(V_{1}, \ldots, V_{N}\right)=\operatorname{det}\left[V_{1}, \ldots, V_{N}\right]
$$

где через $\left[V_{1}, \ldots, V_{N}\right]$ обозначена матрица, составленная из вектор-столбцов $V_{i}$. Обозначим через $\mu\left(\Gamma_{q}\right)$ внешнюю форму степени $N-2$, значение которой на произвольном наборе векторов $V_{1}, \ldots, V_{N-2}$ из $\mathbb{R}^{N}$ определяется так:

$$
\mu\left(\Gamma_{q}\right)\left(V_{1}, \ldots, V_{N-2}\right)=\sum_{p} \omega\left(e_{p}\right) \Theta\left(m_{p}, \nu_{p}, V_{1}, \ldots, V_{N-2}\right),
$$

где сумма берется по всем граничным вершинам $v_{p}$ графа $G_{q}$.

ОПРЕДЕЛЕНИЕ. Внешняя форма $\mu\left(\Gamma_{q}\right)$ называется формой Максвелла невырожденной компоненты $\Gamma_{q}$, или формой Максвелла сети $Г$, соответствуюшей невырожденной компоненте $\Gamma_{q}$. 
ПРЕДЛОЖЕНИЕ 5.8. Пусть $\Gamma_{q}: G_{q} \rightarrow \mathbb{R}^{N}$ - произвольная невырожденная компонента взвешенной минимальной сети Г. Тогда форма Максвелла $\mu\left(\Gamma_{q}\right)$ равна нулю.

ДоКАЗАТЕЛЬСТВо. Для каждой подвижной вершины $v \in G_{q}$ рассмотрим единичные векторы $n_{v}^{j}$ направлений входящих в вершину $\Gamma_{q}: v \rightarrow \mathbb{R}^{N}$ ребер. Так как сеть $\Gamma_{q}$ минимальна, то при каждом фиксированном $v$ линейная комбинация векторов $n_{v}^{j}$ с коэффициентами - весами $\omega_{j}$ соответствующих ребер - равна нулю. Поэтому равна нулю и внешняя $(N-2)$-форма, полученная из формы объема так:

$$
\Theta\left(m_{v}, \sum_{j} \omega_{j} n_{v}^{j}, \cdot, \ldots, \cdot\right)
$$

где через $m_{v}$ обозначен радиус-вектор точки $\Gamma_{q}(v)$. Следовательно, добавив эти равные нулю формы к форме Максвелла $\mu\left(\Gamma_{q}\right)$, мы не изменим форму $\mu\left(\Gamma_{q}\right)$.

Прибавим все эти (нулевые) формы к $\mu\left(\Gamma_{q}\right)$. В полученной сумме мы изменим порядок суммирования, сгруппировав вместе слагаемые, соответствуюшие одному и тому же ребру $e$, а затем уже просуммировав по всем ребрам из $G_{q}$. А именно, пусть $e$ - произвольное ребро из $G, A_{e}$ и $B_{e}$ - радиус-векторы конщевых точек отрезка $\Gamma(e)$, а $n_{A}$ и $n_{B}$ - направления отрезка $\Gamma(e)$, ориентированного соответственно в сторону $A_{e}$ и $B_{e}$. Иными словами, $n_{A}=-n_{B}=B_{e} A_{e} /\left|B_{e} A_{e}\right|$. Тогда ребру $е$ в нашей сумме соответствует следующее выражение:

$$
\begin{aligned}
\omega(e) \Theta\left(A_{e}, n_{A}, \ldots\right)+\omega(e) \Theta\left(B_{e}, n_{B}, \ldots\right) & =\omega(e)\left(\Theta\left(A_{e}, n_{A}, \ldots\right)-\Theta\left(B_{e}, n_{A}, \ldots\right)\right) \\
& =\omega(e)\left(\Theta\left(A_{e}-B_{e}, n_{A}, \ldots\right)\right)=0
\end{aligned}
$$

так как векторы $A_{e}-B_{e}$ и $n_{A}$ коллинеарны. Поэтому форма $\mu\left(\Gamma_{q}\right)$ равна нулю, что и требовалось доказать. Доказательство предложения закончено.

Пусть $G$ - взвешенный графф, совпадаюший со своей невырожденной компонентой. Рассмотрим два граничных отображения $\varphi$ и $\varphi^{\prime}$, одинаковых на всех граничных вершинах из $G$, кроме одной, скажем $v$. Обозначим через $e$ ребро из $G$, инцидентное $v$.

ПРЕДЛОЖЕНИЕ 5.9. Предположим, что $G$ имеет в $\mathbb{R}^{N}$ взвешенные минимальные реализации $\Gamma$ и $\Gamma^{\prime}$ по описанным выше граничным отображениям $\varphi$ и $\varphi^{\prime}$ соответственно. Более того, пусть взвешенные минимальные сети $\Gamma$ и $\Gamma^{\prime}$ параллельны. Тогда отрезок $\left[\varphi(v), \varphi^{\prime}(v)\right]$ параллелен Г-образу ребра е. В частности, отрезки $Г(е) ~ и ~ \Gamma^{\prime}($ е) лежат на одной прямой.

ДокАЗАТЕЛЬСТво. Обозначим через $n$ единичный вектор направлений входящих в вершины $\Gamma: v \rightarrow \mathbb{R}^{N}$ и $\Gamma^{\prime}: v \rightarrow \mathbb{R}^{N}$ ребер $\Gamma: e \rightarrow \mathbb{R}^{N}$ и $\Gamma^{\prime}: e \rightarrow \mathbb{R}^{N}$, а через $m$ и $m^{\prime}-$ радиус-векторы точек $\varphi(v)$ и $\varphi^{\prime}(v)$ соответственно. Рассмотрим формы Максвелла взвешенных минимальных сетей $\Gamma$ и $\Gamma^{\prime}$. Имеем

$$
\mu(\Gamma)-\mu\left(\Gamma^{\prime}\right)=\omega(e) \Theta\left(m-m^{\prime}, n, \ldots\right) .
$$

По предложению 5.8 эта $(N-2)$-форма тождественно равна нулю. Следовательно, вектор $m-m^{\prime}=\varphi^{\prime}(v) \varphi(v)$ коллинеарен вектору $n$. Доказательство предложения закончено. 
5.4. Усы и ядра. Пусть граф $G$ совпадает со своей невырожденной компонентой. Обозначим через $C(G)$ множество всех циклических ребер из $G$. Множество $C(G)$ является подграфом в $G$. Связные компоненты графа $C(G)$ назовем ядрами.

Профакторизуем $G$, как топологическоепространство, по $C(G)$. Результат обозначим через $G^{\prime}$. Полученное факторпространство естественным образом наделяется структурой графа, вершины которого - это вершины из $G$, не входяшие ни в какое ядро, а также все ядра. Две вершины из $G^{\prime}$ смежны тогда и только тогда, когда соответствуюшие им элементы из $G$ (вершины или ядра) соединены в $G$ ребром. Естественную проекцию графа $G$ на факторграф $G^{\prime}$ обозначим через $\pi$. Легко видеть, что $G^{\prime}$ является деревом.

Пусть $\bar{G}$ - произвольное дерево. Для краткости мы будем называть вершины из $\bar{G}$ степени 1 граничнымм так же, как и ребра, инцидентные им. Все остальные ребра будем называть внутренними. Совокупность смежных граничных ребер из $\bar{G}$ назовем усами, если из общей для них вершины выходит не более одного внутреннего ребра. Отметим, что при таком определении усы могут состоять из одного граничного ребра.

ПРЕДЛОЖЕНИЕ 5.10. Каждое дерево $\bar{G}$, количество вериин которого больше единицы, имеет усь. Если это дерево содержит неграничное ребро, то имеются два непересекающихся уса.

ДокаЗАтельство. Отрежем от дерева $\bar{G}$ все граничные ребра. Если у полученного дерева нет ребер, то все граничные ребра из $\bar{G}$ образуют усы. В противном случае у полученного дерева имеется не менее двух вершин степени 1 . Ясно, что все граничные ребра из $\bar{G}$, инцидентные каждой такой вершине степени 1 , образуют усы. Доказательство закончено.

Итак, рассмотрим в факторграфе $G^{\prime}$ некоторые усы. Ясно, что $\pi$-прообраз этих усов - это или набор из $k \geqslant 1$ смежных граничных ребер, или некоторое ядро вместе с выходящими из него граничными ребрами графа $G$. Более того, в последнем случае существует не более одного неграничного нециклического ребра из $G$, инцидентного этому ядру. Полученный $\pi$-прообраз будем называть усами графа $G$, а единственное неграничное нециклическое ребро, инщидентное усам (если таковое имеется) - ребром крепления этих усов. Из предложения 5.10 мгновенно получается следующий результат.

СЛЕДСТВИЕ 5.6. Кажсдий граф с непустой әраницей, совпадающий со своей невырожденной компонентой, имеет некоторые усы. Если этот граф содержит внутреннее нециклическое ребро, то он имеет два непересекающихся yca.

Усы, содержащие циклическое ребро, будем называть ииклическими усами, а все остальные усы - деревянными.

5.5. Доказательство теоремы 5.2. Первое утверждение теоремы вытекает из предложения 5.7. Перейдем к доказательству второго утверждения. Очевидно, его достаточно доказать в предположении, что сеть Г совпадает со своей невырожденной компонентой. Напомним, что в этом случае граница сети - это множество всех ее вершин степени 1. 
Если граф $G$ содержит одно ребро, то утверждение теоремы тривиально. Предположим теперь, что для всех $G$, содержаших менее $n$ ребер, теорема доказана. Пусть $G$ содержит $n$ ребер.

Пусть сначала граф $G$ имеет деревянные усы $\left(e_{1}, \ldots, e_{k}\right), k \geqslant 1$, инщидентные обшей внутренней вершине $v$. Имеется две возможности: или вершина $\Gamma: v \rightarrow \mathbb{R}^{N}$ не является слабо фиктивной в $Г$, или является.

Если вершина $\Gamma: v \rightarrow \mathbb{R}^{N}$ не является слабо фиктивной, то по крайней мере два из отрезков $\Gamma: e_{i} \rightarrow \mathbb{R}^{N}$ не коллинеарны. В силу неизменности направления каждого ребра в процессе деформации $\Gamma_{t}$ вершина $\Gamma: v \rightarrow \mathbb{R}^{N}$ и, значит, все отрезки $\Gamma: e_{i} \rightarrow \mathbb{R}^{N}$ в этом случае неподвижны. По определению усы $\left(e_{1}, \ldots, e_{k}\right)$ состоят из ребер второго уровня, и, как мы только что показали, для них теорема имеет место. Далее, отрежем от $G$ усы $\left(e_{1}, \ldots, e_{k}\right)$ и полученньй невырожденньй графф обозначим через $G^{\prime}$. Обозначим через $\Gamma_{t}^{\prime}$ ограничение деформации $\Gamma_{t}$ на $G^{\prime}$. Так как вершина $\Gamma: v \rightarrow \mathbb{R}^{N}$ неподвижна при деформации $\Gamma_{t}$, то деформация $\Gamma_{t}^{\prime}$ сетей топологии $G^{\prime}$ также неподвижна на гранище. По предположению индукции теорема имеет место для сетей $\Gamma_{t}^{\prime}$. Легко видеть, что ребра из $G^{\prime}$ относятся, по отношению к $G^{\prime}$, к тем же уровням, что и по отношению к $G$. Таким образом, в этом случае теорема доказана.

Пусть теперь вершина $\Gamma: v \rightarrow \mathbb{R}^{N}$ является слабо фиктивной. В этом случае отрезки $\Gamma: e_{i} \rightarrow \mathbb{R}^{N}$ лежат на одной прямой $\ell$, содержащей также ребро крепления $\Gamma: e \rightarrow \mathbb{R}^{N}$ усов. В процессе деформации все эти ребра продолжают лежать на прямой $\ell$, которая, очевидно, остается неподвижной. Поэтому для ребер первого уровня $e, e_{1}, \ldots, e_{k}$ утверждение теоремы имеет место. Пусть $v^{\prime}$ - отличная от $v$ вершина ребра $e$. Ориентируем прямую $\ell$ от $\Gamma\left(v^{\prime}\right)$ к $\Gamma(v)$ и обозначим через $m \in \ell$ такую точку $\Gamma_{t^{\prime}}(v)$, что для всех $t$ точки $\Gamma_{t}(v)$ лежат на замкнутой отрицательной полупрямой $\ell^{\prime} \subset \ell$, ограниченной точкой $m$.

Снова отрежем от графа $G$ усы $\left(e_{1}, \ldots, e_{k}\right)$ и обозначим через $G^{\prime}$ полученный в результате граф. Для каждого $t$ рассмотрим сеть $\Gamma_{t}^{\prime}: G^{\prime} \rightarrow \mathbb{R}^{N}$ такую, что $\left.\Gamma_{t}^{\prime}\right|_{G^{\prime} \backslash e}=\left.\Gamma_{t}\right|_{G^{\prime} \backslash e}$, а $\left.\Gamma_{t}^{\prime}\right|_{e}-$ аффинное отображение, переводящее вершину $v^{\prime}$ в точку $\Gamma_{t}\left(v^{\prime}\right)$, а вершину $v-$ в точку $m$. В силу выбора точки $m$ все $\Gamma_{t}^{\prime}-$ погруженные сети, параллельные $\left.\Gamma_{t}\right|_{G^{\prime}}$. Определим границу графа $G^{\prime}$, положив $\partial G^{\prime}=$ $\left(\partial G \cap G^{\prime}\right) \cup\{v\}$. Граф $G^{\prime}$ с гранищей $\partial G^{\prime}$ совпадает со своей невырожденной компонентой. Далее, положим $\varphi^{\prime}=\partial \Gamma^{\prime}$. По предположению индукции для взвешенной минимальной сети $\Gamma^{\prime}$ утверждение теоремы имеет место. Осталось заметить, что для всех ребер из $G^{\prime}$, кроме ребра $e$, разбиение на уровни по отношению к $G^{\prime}$ совпадает с разбиением на уровни по отношению к $G$. Поэтому для всех этих ребер утверждение теоремы имеет место, что и завершает доказательство.

Пусть теперь в $G$ нет деревянных усов. По следствию 5.6 граф $G$ содержит некоторые циклические усы $G^{\prime}$. Пусть $e$ - ребро крепления усов $G^{\prime}$ и $a \in e-$ некоторая внутренняя точка ребра $e$. Ясно, что ребро $е$ нециклическое. Разрежем граф $G$ по точке $a$. Так как $e$ - нециклическое ребро, граф $G$ распадется на две компоненты. Обозначим через $\bar{G}^{\prime}$ ту из двух компонент, которая содержит усы $G^{\prime}$, а через $e^{\prime}$ - ребро из $\bar{G}^{\prime}$, принадлежащее $e$. Оставшуюся компоненту обозначим через $\bar{G}$.

Рассмотрим ограничение $\Gamma_{t}^{\prime}$ деформации $\Gamma_{t}$ на $\bar{G}^{\prime}$. По предложению 5.9 отрезки $\Gamma_{t}^{\prime}\left(e^{\prime}\right)$ лежат на одной прямой. Поэтому для ребра $e$, которое, очевидно, относится к первому уровню, теорема доказана. Кроме того, мы получаем, что 
для $t$, близких к нулю, можно сделать такую репараметризацию ребер $\Gamma_{t}: e \rightarrow \mathbb{R}^{2}$ сетей $\Gamma_{t}$, которая, во-первых, непрерывно зависит от $t$ и, во-вторых, после нее точка $\Gamma_{t}(a)$ неподвижна. Теперь применим предположение индукции к обеим компонентам $\bar{G}$ и $\bar{G}^{\prime}$. Очевидно, ребра из $\bar{G}$ и $\bar{G}^{\prime}$, отличные от ребер разреза, принадлежат к тем же уровням, что и в графе $G$. Последнее замечание доказывает, что утверждение теоремы имеет место для сетей $\Gamma_{t}$, близких к $\Gamma_{0}=\Gamma$. Применяя описанную процедуру для каждой сети $\Gamma_{t}, t \in[0,1]$, выбранной в качестве сети $\Gamma$, мы получим покрытие отрезка малыми интервалами, на каждом из которых утверждение теоремы выполнено. В силу компактности отрезка из этого покрытия можно выбрать конечное, что и завершает доказательство теоремы.

5.6. Следствия из теоремы 5.2. Теорема 5.2 позволяет описать, чем взвешенные минимальные сети с одинаковыми топологиями и границами отличаются друг от друга.

СлЕДСТВИЕ 5.7. Пусть Г и $\Gamma^{\prime}-$ две взвешенные минимальные сети в $\mathbb{R}^{N}$ одного и того же типа $G$ и с одной и той же границей. Тогда имеют место следующие свойства:

1) сеть Г параллельна сети $\Gamma^{\prime}$;

2) взвешенные длины сетей $Г$ и $\Gamma^{\prime}$ равны;

3) если е - произвольное ребро из $G$, лежащее на втором уровне, то его образы при отображениях $\Gamma и \Gamma^{\prime}$ совпадают;

4) если е- произвольное ребро из $G$, лежащее на первом уровне, то его образы при отображсених $Г$ и $\Gamma^{\prime}$ лежсат на одной прямой;

5) если е - произвольное ребро из $G$, лежащее на нулевом уровне, то его образы при отображсениях $\Gamma$ и $\Gamma^{\prime}$ (в соответствии со свойством 1) параллельны межсду собой.

Следствие 5.7 описывает, чем могут отличаться друг от друга соответствуюшие ребра двух взвешенных минимальных сетей одного типа с одинаковой границей. Изучим теперь, чем могут отличаться соответствуюшие вершины таких сетей. Для этого разобьем вершины параметризующего графа $G$ на уровни так. $\mathrm{K}$ нулевому уровню отнесем все (подвижные) вершины графа $G$, которые инцидентны только ребрам нулевого уровня. $\mathrm{K}$ первому уровню отнесем те подвижные вершины графа $G$, не попавшие на нулевой уровень, которые не иншидентны ребрам второго уровня. Все остальные вершины графа $G$ отнесем ко второму уровню. Для каждой вершины $v$ графа $G$ и каждого граничного отображения $\varphi$ назовем локусом вершины $v$ для отображения $\varphi$ замыкание подмножества пространства $\mathbb{R}^{N}$, состояшего из точек вида $\Gamma(v)$ по всем локально минимальным (взвешенным минимальным) сетям $\Gamma$ типа $G$ с границей $\varphi$. Локус вершины $v$ для граничного отображения $\varphi$ графа $G$ обозначим через $\operatorname{loc}_{\varphi}(v)$.

СЛЕДСТВИЕ 5.8. В сделанных выше обозначениях если $v$-вершина второго уровня, то ее локус состоит из одной точки. Локус вериин первого и нулевого уровней представляет собой выпуклый многогранник в $\mathbb{R}^{N}$, размерность которого не превосходит $\min \{N, m-r\}$, а количество граней максимальной размерности не превосходит $\mathrm{m}$. 


\section{§ 6. Взвешенные минимальные сети Штейнера на плоскости}

Рассмотрим теперь важный частный случай - плоские сети, степени вершин которых не превосходят трех. Такие сети и их параметризующие графы называются плоскими сетями Штейнера и графами Штейнера соответственно. Они возникают при исследовании локально минимальных сетей в смысле функционала обычной (не взвешенной) длины: каждая локально минимальная сеть есть сеть Штейнера. Для плоских взвешенных минимальных сетей Штейнера, не содержащих слабо фиктивных вершин степени 3 , удается вычислить размерность многогранника $[G, \varphi]_{\min }$, исходя из топологии параметризующего графа $G$ с гранищей $\partial G$. Оказывается, ответ полностью аналогичен полученному в [47] результату для обычных локально минимальных сетей: искомая размерность равна сумме цикломатического числа подвижного подграфа графа $G$ и количества фиктивных вершин граффа $G$.

Отметим, что наше предположение об отсутствии у взвешенных минимальных сетей слабо фиктивных вершин степени 3 равносильно тому, что в каждой внутренней вершине $v$ степени 3 рассматриваемых взвешенных графов Штейнера неравенство треугольника для весов инцидентных $v$ ребер выполняется строго.

6.1. Взвешенная минимальная реализация плоских сетей. Пусть $G$ произвольный взвешенньй граф Штейнера, $\partial G$ - некоторая его граница, и пусть $\varphi: \partial G \rightarrow \mathbb{R}^{2}$ - произвольное граничное отображение. Мы говорим, что $G$ имеет взвешенную минимальную реализацию с әраницей $\varphi$, если существует (погруженная) взвешенная минимальная сеть $\Gamma: G \rightarrow \mathbb{R}^{2}$ с границей $\partial \Gamma$, равной $\varphi$. Иногда, там где это не вызовет недоразумений, мы будем называть взвешенную минимальную реализацию просто минимальной реализачией, опуская слово “взвешенная".

ЗАмЕчАниЕ. Пусть задано некотороеграничное отображение $\varphi: \partial G \rightarrow \mathbb{R}^{2}$ границы $\partial G$ графа $G$. В дальнейшем мы, для удобства, будем обозначать подвижный подграф $\widehat{G}_{\partial G}$ графо $G$ по отношению к границе $\partial G$ через $\widehat{G}_{\varphi}$ и назьвать его подвижным подграфом графа $G$ по отношению к граничному отображению $\varphi$.

Если $G$ - дерево Штейнера, то имеется придуманное авторами обобщение знаменитого алгоритма Мелзака [20], позволяющее понять, имеет ли данное взвешенное дерево Штейнера взвешенную минимальную реализацию с заданной границей $\varphi$, и если имеет, то построить соответствующую взвешенную минимальную сеть (см. [42], [36]).

Напомним этот алгоритм.

6.2. Обобщенный алгоритм Мелзака для взвешенных деревьев Штейнера. Пусть $G$ - взвешенное дерево Штейнера с весовой функцией $\omega$ и границей $\partial G$ и $\varphi: \partial G \rightarrow \mathbb{R}^{2}-$ некоторое граничное отображение. Из сказанного выше вытекает, что мы можем ограничиться случаем невырожденных деревьев Штейнера без фиктивных вершин, т.е. бинарных деревьев (или 2-деревьев), границы которых состоят из всех вершин степени 1.

Если $G$ имеет ровно одно ребро, то, очевидно, взвешенная минимальная реализация сушествует тогда и только тогда, когда образы граничных вершин этого дерева различны (напомним, что в соответствии с нашим соглашением, все ребра сети - вложенные кривые). Пусть теперь $G$ состоит более чем из одного ребра. 


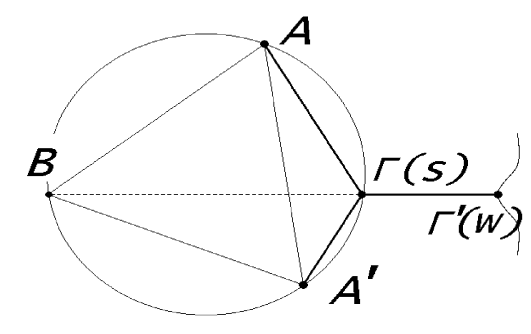

Рис. 1. Иллюстрация идеи обобщенного алгоритма Мелзака

Выше мы определили понятие усов для произвольного дерева. В рассматриваемом случае, очевидно, имеются две возможности: или 2-дерево $G$ не содержит внутренних ребер (т.е. состоит из одного или трех граничных ребер) и совпадает со своими единственными усами, или дерево $G$ содержит некоторое внутреннее ребро, и тогда любые усы состоят из пары смежных граничных ребер. В последнем случае дерево $G$ имеет не менее двух непересекаюшихся усов.

Чтобы не рассматривать отдельно случай 2-дерева с тремя ребрами, мы переопределим понятие усов в этом случае. А именно, назовем усами произвольного бинарного дерева любую пару смежных граничных ребер. При таком определении для 2-деревьев, содержаших внутренние ребра, понятие усов не меняется, а для бинарного дерева из трех ребер каждая пара его ребер образует усы. (В последнем случае каждые два уса пересекаются.)

Итак, 2-дерево $G$, состоящее более чем из одного ребра, имеет некоторые усы $\left(e, e^{\prime}\right)$. Пусть $v$ и $v^{\prime}$ - вершины степени 1 , инцидентные ребрам $e$ и $e^{\prime}$ соответственно, а $s$ - вершина степени 3 , инщидентная одновременно ребрам $e$ и $e^{\prime}$. Обозначим через $e^{\prime \prime}$ третье ребро, инцидентное вершине $s$, а через $w$-отличную от $s$ вершину, инцидентную ребру $e^{\prime \prime}$.

Если образы $A=\varphi(v)$ и $A^{\prime}=\varphi\left(v^{\prime}\right)$ вершин $v$ и $v^{\prime}$ при отображении $\varphi$ совпадают, то взвешенной минимальной реализации не сушествует. В противном случае построим одним из двух возможных способов треугольник $A A^{\prime} B$ такой, что $|A B|=\left|A A^{\prime}\right| \omega\left(e^{\prime}\right) / \omega\left(e^{\prime \prime}\right)$ и $\left|A^{\prime} B\right|=\left|A A^{\prime}\right| \omega(e) / \omega\left(e^{\prime \prime}\right)$. Так как веса $\omega(e)$, $\omega\left(e^{\prime}\right)$ и $\omega\left(e^{\prime \prime}\right)$ удовлетворяют правилу треугольника, то такой треугольник $A A^{\prime} B$ существует.

Перестроим дерево $G$ в дерево $G^{\prime}$, отрезав от $G$ усы. Определим граничное отображение $\varphi^{\prime}$ на эффективной гранище $\partial G^{\prime}$ дерева $G^{\prime}$, положив его равным $\varphi$ везде, кроме $s$, и определив $\varphi^{\prime}(s)$ равным $B$. Наконец определим индуцированную весовую функцию $\omega^{\prime}$ на ребрах из $G^{\prime}$ равной $\omega$.

Следующая лемма вытекает из элементарных планиметрических построений.

Лемма 6.1. Если взвешенное 2-дерево $G$ с весовой функиией $\omega$ имеет минимальную реализацию $Г$ с границей $\varphi$, то для одного из двух треугольников $A A^{\prime} B$, определенных выше, взвешенное дерево $G^{\prime}$ с весовой функиией $\omega^{\prime}$ имеет минимальную реализацию $\Gamma^{\prime} c$ граничей $\varphi^{\prime}$. Взвешенные минимальнье сети Г и $\Gamma^{\prime}$ совпадают на $G^{\prime} \backslash e^{\prime \prime}$. При әтом луи с верииной $B$ в направлении точки $\Gamma^{\prime}(w)=\Gamma(w)$ содержится внутри угла $A B A^{\prime}$ и содержит точку $Г(s)$, которая, в свою очередь, лежит на окружности $S^{1}$, описанной 
вокруг треугольника $A B A^{\prime} . B$ частности, точка $\Gamma^{\prime}(w)=\Gamma(w)$ лежит вне круга, ограниченного окружностью $S^{1}$.

Обратно, если существует минимальная реализация $\Gamma^{\prime}$ взвешенного дерева $G^{\prime}$ с границей $\varphi^{\prime}$ и выполняются следующие условия:

1) луч с вершиной $B$ в направлении точки $\Gamma^{\prime}(w)$ содержится внутри угла $A B A^{\prime}$,

2) точка $\Gamma^{\prime}(w)$ лежст вне круга, ограниченного окружностью $S^{1}$, которая описана вокруг треугольника $A B A^{\prime}$,

то существует минимальная реализация $Г$ взвешенного дерева $G$ с границей $\varphi$, которая получается из $\Gamma^{\prime}$ так. Обозначим через $C$ точку пересечения интервала $\left(\Gamma^{\prime}(s), \Gamma^{\prime}(w)\right)$ с окружностью $S^{1}$. На всех ребрах из $G$, отличных от e, $e^{\prime}$ и $e^{\prime \prime}$, отображение Г определим равным отображению $\Gamma^{\prime}$. На ребрах e, $e^{\prime}$ и $e^{\prime \prime}$ определим его так, чтобъ эти ребра переходили соответственно в отрезки $[C, A],\left[C, A^{\prime}\right] u\left[C, \Gamma^{\prime}(w)\right]$. При этом конечно же $\Gamma(s)=C$.

Итерируя описанный только что процесс перестройки взвешенного дерева $G$ и граничного отображения $\varphi$ во взвешенное дерево $G^{\prime}$ и граничное отображение $\varphi^{\prime}$ до тех пор, пока в результируюшем дереве останется ровно одно ребро, мы реализуем прямой ход обобщенного алгоритма Мелзака. Каждая итерация называется шагом прямого хода обобщенного алгоритма Мелзака. Ясно, что если прямой ход состоит из $n$ шагов, то сушествует $2^{n}$ реализаций прямого хода, в зависимости от выбора треугольника $A B A^{\prime}$ на каждом шаге.

Для завершения обобшенного алгоритма Мелзака необходимо выполнить обратный ход. На первом шаге обратного хода мы проверяем, не совпали ли образы граничных вершин результирующего дерева, состоящего из одного ребра. Если не совпали, то строим взвешенную минимальную реализацию этого дерева, отображая единственное его ребро в отрезок, соединяющий образы граничных вершин при результирующем граничном отображении. После этого начинаем последовательно строить минимальные реализации взвешенных деревьев, полученных на прямом ходе. Соответствуюшие построения мы уже описали в лемме 6.1. Если на одном из шагов обратного хода нарушаются условия леммы 6.1 , то переходим к испытанию следующей из $2^{n}$ реализаций прямого хода. Таким образом, или при проверке очередной реализации прямого хода обратный ход обобщенного алгоритма Мелзака приведет к построению искомой взвешенной минимальной реализации, или будут проверены все $2^{n}$ вариантов и сделан вывод о том, что данное взвешенное дерево $G$ не имеет минимальной реализации с граничным отображением $\varphi$.

ЗАмЕЧАнИЕ. Из предложения 5.6 вытекает, что к успешному завершению прямого хода обобщенного алгоритма Мелзака может привести не более одной реализации прямого хода. Обобщенный алгоритм Мелзака, не отсеивая "неперспективные" последовательности, тратит много времени на работу с ними. Однако оказывается, в случае, когда все веса одинаковы (фактически когда $\ell_{\omega}-$ функционал длины), можно заранее понять, как устроена та единственная последовательность треугольников $A B A^{\prime}$, которая может привести к успешному завершению обобщенного алгоритма Мелзака. Эту задачу решает алгоритм, предложенный Хвангом в [15]. В настоящее время авторам не известно, можно ли обобщить алгоритм Хванга на случай взвешенных бинарных деревьев. 
ЗАмечАниЕ. Ясно, что алгоритм Мелзака применим также и к случаю невырожденного дерева Штейнера с фиктивными вершинами. А именно, достаточно провести описанные вьше построения для невырожденного дерева, полученного из исходного выбрасыванием всех фиктивных вершин, а затем добавить фиктивные вершины в произвольные места соответствуюших ребер.

6.3. Следствия из обобщенного алгоритма Мелзака. Как было отмечено выше, для успешного завершения обратного хода обобщенного алгоритма Мелзака необходимо и достаточно выполнение на каждом шаге условий леммы 6.1. Легко видеть, что если существует минимальная реализация взвешенного невырожденного дерева Штейнера $G$ с граничным отображением $\varphi$, то при малом шевелении границы, т.е. при малом изменении граничного отображения $\varphi$, условия леммы 6.1 по-прежнему выполняются. Чтобы сформулировать соответствуюший результат, введем следующее определение.

Пусть $G$ - произвольный граф с границей $\partial G$. Введем на множестве всех граничных отображений $\varphi: \partial G \rightarrow \mathbb{R}^{2}$ метрику $\rho$ следующим образом. Если $\varphi$ и $\varphi^{\prime}-$ два таких отображения, то положим

$$
\rho\left(\varphi, \varphi^{\prime}\right)=\max _{v \in \partial G}\left|\varphi(v), \varphi^{\prime}(v)\right| .
$$

ПРЕДЛОЖЕнИЕ 6.1. Предположим, что взвешенное дерево Штейнера G c граничей $\partial G$ имеет минимальную реализачию Г для некоторого граничного отображения $\varphi$. Тогда существует такое $\varepsilon>0$, что для любого граничного отображения $\varphi^{\prime}, \rho\left(\varphi, \varphi^{\prime}\right)<\varepsilon$, взвешенное дерево $G$ также имеет минимальную реализачию с граничным отображсением $\varphi^{\prime}$. Иными словами, взвешенные минимальные деревья Штейнера устойчивы при малых вариациях границы.

Более того, для любого $\varepsilon>0$ существует такое $\delta>0$, что для всех граничных отображсений $\varphi^{\prime}$ дерева Штейнера $G$ таких, что $\rho\left(\varphi^{\prime}, \varphi\right)<\delta$, существуют минимальные реализачии $\Gamma^{\prime}$ взвешенного дерева $G$ с границами $\varphi^{\prime}$ и расстояние между образами $\Gamma(v)$ u $\Gamma^{\prime}(v)$ произвольной вериины $v \in G$ при отображениях $\Gamma$ и $\Gamma^{\prime}$ меньше $\varepsilon$. Иными словами, минимальная реализачия взвешенного дерева Штейнера $G$ непрерывно зависит от граничного отображения этого дерева.

Напомним, что результатом прямого хода обобщенного алгоритма Мелзака является пара точек. В качестве первого шага обратного хода мы строим отрезок, соединяющий полученные точки. Этот отрезок называется линией Симпсона. Отметим, что в силу произвольности выбора усов на каждом шаге прямого хода мы, вообще говоря, можем построить много разных линий Симпсона. На самом деле если $G$ - бинарное дерево, то существует естественное взаимно однозначное соответствие между линиями Симпсона и ребрами дерева $G$. Действительно, в процессе прямого хода мы перестраиваем дерево $G$, отрезая от него выбранные усы. На последнем шаге прямого хода от дерева $G$ остается ровно одно ребро, которое и надо поставить в соответствие полученной линии Симпсона. Несложно показать, что каждому ребру дерева $G$ соответствует некоторая линия Симпсона.

Если же $G$ - невырожденное дерево Штейнера с фиктивными вершинами, то каждому его ребру соответствует ребро бинарного дерева, полученного из $G$ выбрасыванием из $G$ всех фиктивных вершин и, значит, некоторая линия Симпсона. 
Однако теперь взаимно однозначного соответствия нет: ребрам из $G$, инщидентньм одной и той же фиктивной вершине, соответствует одна и та же линия Симпсона. Отметим, что эти ребра обязаны иметь одинаковые веса.

Имеет место следуюшее утверждение (см. [42], [36]).

УТВЕРЖДЕНИЕ 6.1. Если взвешенное невырожденное дерево Штейнера $G$ c весовой функцией $\omega$ имеет минимальную реализачию $Г$ с граничным отображсением $\varphi$, то длина линии Симпсона, соответствующей ребру е $\in E(G)$, равна $\ell_{\omega}(\Gamma) / \omega($ e). Иными словами, взвешенная длина линии Симпсона, т.е. длина этой линии, умноженная на вес соответствующего ей ребра, равна взвешенной длине сети Г.

В следующем пункте мы покажем, как можно легко вычислить длину линии Симпсона, исходя только из информации о структуре погружения, задающего взвешенное минимальное невырожденное дерево Штейнера, его весовой функции и координатах граничных точек. Мы начнем с того, что опишем важную характеристику погружения, а именно оснащение вращения, индуцированное этим погружением.

6.4. Оснащение вращения. Пусть $G$ - произвольный граф̆ и $\Gamma: G \rightarrow \mathbb{R}^{2}-$ некоторая сеть. Напомним, что по определению ребра сети - вложенные кривые. Тем не менее, минимальные взвешенные сети обладают еще одним важным свойством: у каждой вершины такой сети имеется вложенная локальная сеть (напомним, что мы предполагаем выполненными строгие неравенства треугольника для весов). Каждую сеть, обладающую этим дополнительным свойством, будем называть нормальной.

Пусть теперь $\Gamma$ - нормальная невырожденная сеть Штейнера. Отметим, что так как Г невырождена, т.е. ее граница состоит в точности из всех вершин степени 1 , то множество всех подвижных вершин сети $\Gamma$ - это множество всех вершин из $Г$ степени 2 и 3 , причем каждая вершина степени 2 фиктивная.

Пусть $v$-произвольная подвижная вершина из Г степени 3 . В силу нормальности сети Г некоторая локальная сеть $\Gamma_{U}$ с центром в $v$ является вложенной сетью, состоящей из трех ребер. Пусть $е$ и $e^{\prime}-$ произвольные два ребра из $G_{U}$. Положим по определению число вращения $\mathrm{tw}\left(e, e^{\prime}\right)$ упорядоченной пары $\left(e, e^{\prime}\right)$ равньм 1 , если при обходе в положительном направлении вершины $v$, начиная с образа ребра $e^{\prime}$, мы сначала встретим образ ребра $e$, а лишь затем образ третьего ребра сети $\Gamma_{U}$. $\mathrm{B}$ противном случае положим $\operatorname{tw}\left(e, e^{\prime}\right)=-1$. Если $E$ и $E^{\prime}-$ peбра графа $G$, coдержащие $е$ и $e^{\prime}$ соответственно, то числом вращения $\operatorname{tw}\left(E, E^{\prime}\right)$ упорядоченной пары $\left(E, E^{\prime}\right)$, индуцированным сетью $\Gamma$, назовем число tw $\left(e, e^{\prime}\right)$.

Пусть теперь $v$ - произвольная фиктивная вершина графа $G$, а $E$ и $E^{\prime}$ - ребра из $G$, инцидентные $v$. Положим по определению $\operatorname{tw}\left(E, E^{\prime}\right)=0$. Итак, каждая нормальная сеть $\Gamma: G \rightarrow \mathbb{R}^{2}$ индуцирует на $G$ оснащение вращения, представляющее собой совокупность чисел вращения всех упорядоченных пар смежных ребер из $G$.

Пусть теперь $G$ - взвешенный невырожденный граф Штейнера с весовой функцией $\omega$ и $\Gamma$ - некоторая нормальная сеть типа $G$, а $v$ - произвольная подвижная вершина графа $G$ степени 3 , инцидентная ребрам $E_{i}, i=1,2,3$. Построим треугольник со сторонами, равными весам $\omega\left(E_{i}\right)$, и обозначим через $\alpha_{i}$ величину того 
угла этого треугольника, который противолежит стороне длины $\omega\left(E_{i}\right)$. Положим

$$
\mathrm{tw}^{\omega}\left(E_{i}, E_{j}\right)=\frac{3 \alpha_{k}}{\pi} \operatorname{tw}\left(E_{i}, E_{j}\right)
$$

где $\{i, j, k\}=\{1,2,3\}$. Число tw ${ }^{\omega}\left(E_{i}, E_{j}\right)$ называется взвешенным числом вращения упорядоченной пары $\left(E_{i}, E_{j}\right)$ ребер взвешенного графа $G$, индуцированньм нормальной сетью $Г$.

Пусть теперь $v$-произвольная фиктивная вершина из $G$, а $E$ и $E^{\prime}$ - инцидентные ей ребра из $G$. Положим tw ${ }^{\omega}\left(E, E^{\prime}\right)=0$. Итак, каждая взвешенная невырожденная нормальная сеть Штейнера $\Gamma: G \rightarrow \mathbb{R}^{2}$ индуцирует на $G$ взвешенное оснащение вращения, представляюшее собой совокупность взвешенных чисел вращения всех упорядоченных пар смежных ребер из $G$.

Если $G$ является невырожденным деревом Штейнера, то можно продолжить как оснашение вращения, так и взвешенное оснащение вращения на все упорядоченные пары ребер этого дерева по аддитивности вдоль путей. Последнее означает следуюшее. Если $\left(E, E^{\prime}\right)$ - упорядоченная пара произвольных различных ребер из $G$, а $\gamma=\left\{E_{0}=E, E_{1}, \ldots, E_{k}=E^{\prime}\right\}$ - единственный путь в $G$, соединяющий $E$ с $E^{\prime}$, то положим

$$
\operatorname{tw}\left(E, E^{\prime}\right)=\sum_{p=1}^{k} \operatorname{tw}\left(E_{p-1}, E_{p}\right), \quad \operatorname{tw}^{\omega}\left(E, E^{\prime}\right)=\sum_{p=1}^{k} \mathrm{tw}^{\omega}\left(E_{p-1}, E_{p}\right)
$$

Если же $E=E^{\prime}$, то положим

$$
\operatorname{tw}\left(E, E^{\prime}\right)=\operatorname{tw}^{\omega}\left(E, E^{\prime}\right)=0 .
$$

Число tw $\left(E, E^{\prime}\right)$ называется числом вращения упорядоченной пары $\left(E, E^{\prime}\right)$ ребер дерева $G$, или числом вращения от ребра $E \kappa$ ребру $E^{\prime}$, порожденньм сетью Г. Соответственно число tw ${ }^{\omega}\left(E, E^{\prime}\right)$ назьвается взвешеннылм числом вращения упорядоченной пары $\left(E, E^{\prime}\right)$ ребер взвешенного дерева $G$, или взвешенным числом вращения от ребра $E \kappa$ ребру $E^{\prime}$, порожденным взвешенной сетью $Г$.

Отметим, что если Г - взвешенное минимальное невырожденное дерево Штейнера, то взвешенное число врашения имеет естественный геометрический смысл. Чтобы сформулировать соответствующее утверждение, напомним следующие понятия.

Пусть $(a, b)$ - упорядоченная пара ненулевых векторов из $\mathbb{R}^{2}$. Определим ориентированный угол $\alpha(a, b)$ nары $(a, b)$ следующим образом. Если $a$ и $b$ сонаправлены, то положим $\alpha(a, b)=0$. Если $a$ и $b$ не коллинеарны, то определим $\alpha(a, b)$ равным по модулю наименьшему углу между $a$ и $b$, а по знаку - знаку репера $(a, b)$. Если же $a$ и $b$ противоположно направлены, то ориентированный угол $\alpha(a, b)$ не определен.

Далее, погруженной ломаной $L$ на плоскости назовем последовательность точек $A_{0}, \ldots, A_{k}$ таких, что все отрезки $a_{p}$ вида $\left[A_{p-1}, A_{p}\right]$ невырождены, а последовательные отрезки $a_{p}=\left[A_{p-1}, A_{p}\right]$ и $a_{p+1}=\left[A_{p}, A_{p+1}\right]$ пересекаются лишь по вершине $A_{p}$. Точки $A_{p}$ называются вершинами ломаной $L$, а отрезки $a_{p}-p e б р а-$ мu (или звеньями) ломаной $L$. 
Вершины $A_{0}, \ldots, A_{k}$ ломаной $L$ можно упорядочить двумя естественными способами: от $A_{0}$ к $A_{k}$ и от $A_{k}$ к $A_{0}$. Выбор одного из этих порядков называется ориентацией ломаной $L$, а сама ломаная с фиксированным порядком вершин ориентированной ломаной. При этом если вершины $A_{p}$ упорядочены от $A_{0} \mathrm{~K} A_{k}$, то мы говорим, что ломаная $L$ ориентирована от $A_{0} \kappa A_{k}$ (или от $a_{1} \kappa a_{k}$ ). В противном случае ломаная $L$ ориентирована от $A_{k} \kappa A_{0}$ (или om $a_{k} \kappa a_{1}$ ).

Для ориентированной ломаной $L$ каждое ее звено $a_{p}=\left[A_{p}, A_{p+1}\right]$ можно рассматривать как вектор $A_{p} A_{p+1}$ с началом в $A_{p}$. Такие векторы будем называть вектор-звеньями.

Углом поворота между последовательными вектор-звеньями $a_{p}$ и $a_{p+1}$ ориентированной ломаной $L$ назьвается угол $\alpha\left(a_{p}, a_{p+1}\right)$. Сумма углов поворота между всеми последовательными ребрами $a_{p}$ и $a_{p+1}$ ориентированной ломаной $L$ называется полным углом поворота вдоль ломаной $L$ и обозначается через $\alpha(L)$.

Пусть теперь $\left(a, a^{\prime}\right)$ - упорядоченная пара звеньев произвольной (неориентированной) ломаной $L$. Рассмотрим ломаную $L^{\prime}$, составленную из всех звеньев ломаной $L$, расположенных между $a$ и $a^{\prime}$. Ориентируем $L^{\prime}$ от $a$ к $a^{\prime}$. Углом поворота упорядоченной пары $\left(a, a^{\prime}\right)$ ребер ломаной $L$ (или углом поворота от ребра а $\kappa$ ребру $a^{\prime}$ ломаной $\left.L\right)$ называется полный угол поворота ломаной $L^{\prime}$.

Пусть теперь $G$ - взвешенное невырожденное дерево Штейнера и $\Gamma$ - некоторая его минимальная реализация. Как уже было отмечено выше, погружение Г нормальное, поэтому на $G$ индуцируется некоторое взвешенное оснашение вращения. Выберем в $G$ два произвольных ребра $e$ и $e^{\prime}$, и пусть $\gamma$ - единственный путь в $G$, соединяющий $e$ c $e^{\prime}$. Образ пути $\gamma$ при отображении Г задает некоторую погруженную ломаную $L$. Ориентируем $L$ от образа ребра $e$ к образу ребра $e^{\prime}$. Имеет место следуюший очевидный результат.

ПРЕДЛОЖЕНИЕ 6.2. Взвешенное число вращения tw ${ }^{\omega}\left(e, e^{\prime}\right)$ упорядоченной пары $\left(e, e^{\prime}\right)$ равно полному углу поворота $\alpha(L)$ вдоль ломаной $L$, деленному на $\pi / 3$ :

$$
\operatorname{tw}^{\omega}\left(e, e^{\prime}\right)=\frac{3}{\pi} \alpha(L) .
$$

Далее, в терминах чисел вращения легко выписать координаты конщов линии Симпсона. Пусть $G$ - произвольное взвешенное невырожденное дерево Штейнера и $\Gamma$ - некоторая его взвешенная минимальная реализация с эффективной границей $\partial Г$. Выберем в $G$ произвольное ребро $e$, и пусть $L=[X, Y]$ - соответствующая е линия Симпсона.

Пусть ребро е граничное, $v$ - единственная граничная вершина, ему инцидентная, и $m=\Gamma(v)$ - ее образ при отображении $\Gamma$. Тогда, как легко видеть, одна из концевых точек линии $L$, скажем $X$, совпадает с $m$. Далее, пусть $v_{1}, \ldots, v_{k}-$ остальные граничные вершины из $G$, а $m_{p}=\Gamma\left(v_{p}\right)$. Пусть $e_{p}$ - единственное граничное ребро, инщидентное $v_{p}$. Обозначим через $t_{p}$ взвешенное число врашения $\mathrm{tw}^{\omega}\left(e, e_{p}\right)$. Тогда имеет место следующее предложение.

ПРЕДЛОЖЕНИЕ 6.3. Второй конеи $Y$ линии Cимпсона L может быть вычислен по следующей формуле:

$$
Y=\frac{1}{\omega(e)} \sum_{p=1}^{k} \omega\left(e_{p}\right) m_{p} e^{-i t_{p} \pi / 3}
$$

где через $m_{p}$ обозначены комплексные координаты точек $m_{p}$. 
ДоКАЗАТЕЛЬСТво. Для доказательства этого предложения нам понадобится следующая лемма.

ЛЕмма 6.2. Пусть $A B C$ - треугольник на плоскости $\mathbb{R}^{2}$ со сторонами $a=|B C|, \quad b=|A C|, \quad c=|A B|$ и углами $\alpha=\widehat{A}, \beta=\widehat{B}, \gamma=\widehat{C}$. Предположим, что пара векторов $(A B, A C)$ образует положительно ориентированный репер. Тогда вершина $C$ может быть вычислена через $A$ и $B$ так:

$$
c C=a A e^{-i \beta}+b B e^{i \alpha} .
$$

ДокаЗАТЕЛЬСТво. В самом деле,

$$
C=A+\frac{b(B-A) e^{i \alpha}}{c}
$$

поэтому

$$
c C=A\left(c-b e^{i \alpha}\right)+b B e^{i \alpha} .
$$

$\mathrm{C}$ другой стороны, сумма векторов $A B, B C$ и $C A$ равна нулю, что на комплексном языке переписывается так:

$$
c+a e^{i(\pi-\beta)}+b e^{-i(\pi-\alpha)}=0
$$

откуда

$$
c-b e^{i \alpha}=a e^{-i \beta},
$$

что и требовалось доказать.

Отметим сначала, что по определению линии Симпсона для невырожденного дерева Штейнера совпадают с соответствующими линиями Симпсона для бинарного дерева, полученного выбрасыванием фиктивных вершин. Поэтому доказательство предложения достаточно провести в предположении, что $G$ - бинарное дерево.

Итак, пусть $G$ - бинарное дерево. Доказательство проведем по индукции. Если дерево состоит из одного ребра $e$, образ которого - отрезок $[A, B]$, то линия Симпсона совпадает с этим отрезком. С другой стороны, если $X=A$, то по доказываемой формуле второй конец $Y$ линии Симпсона должен иметь вид:

$$
Y=\frac{1}{\omega(e)} \omega(e) B=B
$$

что верно.

Далее, предположим, что для всех бинарных деревьев, содержаших меньше чем $n$ вершин степени 1 , предложение доказано. Пусть теперь бинарное дерево $G$ содержит $n \geqslant 3$ вершин степени 1 .

По предложению 5.10 дерево $G$ имеет усы $\left(e^{\prime}, e^{\prime \prime}\right)$, не содержащие ребра $e$. Пусть $f$ - единственное ребро из $G$, смежное одновременно с $e^{\prime}$ и $e^{\prime \prime}$. Без ограничения общности будем считать, что $\operatorname{tw}^{\omega}\left(f, e^{\prime}\right)>0$, поэтому, в частности, $\operatorname{tw}^{\omega}\left(f, e^{\prime \prime}\right)<0$.

Обозначим через $A$ и $B$ образы вершин степени 1 , инцидентных соответственно $e^{\prime}$ и $e^{\prime \prime}$, при отображении $\Gamma$. Применим к сети $Г$ шаг прямого хода обобшенного алгоритма Мелзака, заменив вершины $A$ и $B$ на вершину $C$ такую, что длины 
сторон $a=|B C|, \quad b=|A C|$ и $c=|A B|$ треугольника $A B C$ равны соответственно $\lambda \omega\left(e^{\prime}\right), \lambda \omega\left(e^{\prime \prime}\right)$ и $\lambda \omega(f)$ для некоторого $\lambda>0$, а векторы $A B$ и $A C$ образуют положительно ориентированный репер. Обозначим через $\alpha$ и $\beta$ величины углов треугольника $A B C$ в вершинах $A$ и $B$. Отметим, что в соответствии с геометрическим смыслом взвешенного числа врашения имеем $\alpha=-\operatorname{tw}^{\omega}\left(f, e^{\prime \prime}\right) \pi / 3$, a $\beta=\operatorname{tw}^{\omega}\left(f, e^{\prime}\right) \pi / 3$.

Пусть $\Gamma^{\prime}: G^{\prime} \rightarrow \mathbb{R}^{2}$ - результат работы описанного только что шага алгоритма и $w$ - вершина из $G^{\prime}$, соответствуюшая $C$. Положим $t=\operatorname{tw}^{\omega}(e, f)$. Тогда для $\Gamma^{\prime}$ предложение справедливо по предположению индукции. Иными словами,

$$
Y=\frac{1}{\omega(e)} \sum_{p: v_{p} \in G^{\prime}} \omega\left(e_{p}\right) m_{p} e^{-i t_{p} \pi / 3}+\frac{1}{\omega(e)} \omega(f) C e^{-i t \pi / 3}
$$

(отметим, что линия Симпсона сохраняется при работе обобшенного алгоритма Мелзака). Однако последнее слагаемое в этом выражении в силу леммы 6.2 можно переписать в виде

$$
\begin{aligned}
\frac{1}{\omega(e)} \omega & (f) C e^{-i t \pi / 3}=\frac{1}{\lambda \omega(e)} \lambda \omega(f) C e^{-i t \pi / 3} \\
& =\frac{1}{\lambda \omega(e)}\left(\lambda \omega\left(e^{\prime}\right) A e^{-i \beta}+\lambda \omega\left(e^{\prime \prime}\right) B e^{i \alpha}\right) e^{-i t \pi / 3} \\
& =\frac{1}{\omega(e)}\left(\omega\left(e^{\prime}\right) A e^{-i \mathrm{tw}^{\omega}\left(f, e^{\prime}\right) \pi / 3}+\omega\left(e^{\prime \prime}\right) B e^{-i \mathrm{tw}^{\omega}\left(f, e^{\prime \prime}\right) \pi / 3}\right) e^{-i t \pi / 3} \\
& =\frac{1}{\omega(e)}\left(\omega\left(e^{\prime}\right) A e^{-i \mathrm{tw}^{\omega}\left(e, e^{\prime}\right) \pi / 3}+\omega\left(e^{\prime \prime}\right) B e^{-i \mathrm{tw}^{\omega}\left(e, e^{\prime \prime}\right) \pi / 3}\right)
\end{aligned}
$$

откуда и вытекает требуемое утверждение. Предложение доказано.

Далее, пусть теперь $e$ - некоторое неграничное ребро дерева $G$. Разрезав $G$ по ребру $e$, мы разобьем дерево $G$ на два невырожденных дерева Штейнера, скажем $G^{\prime}$ и $G^{\prime \prime}$. Обозначим через $m_{1}^{\prime}, \ldots, m_{k^{\prime}}^{\prime}$ вершины степени 1 из $G^{\prime}$, не принадлежашие $e$, а через $m_{1}^{\prime \prime}, \ldots, m_{k^{\prime \prime}}^{\prime \prime}$ - вершины степени 1 из $G^{\prime \prime}$, также не принадлежащие $e$. Пусть $e_{p}^{\prime}$ - ребро из $G$, инцидентное $m_{p}^{\prime}$, а $e_{p}^{\prime \prime}$ - ребро из $G$, инцидентное $m_{p}^{\prime \prime}$. Обозначим через $t_{p}^{\prime}$ и $t_{p}^{\prime \prime}$ числа вращения $\mathrm{tw}^{\omega}\left(e, e_{p}^{\prime}\right)$ и $\mathrm{tw}^{\omega}\left(e, e_{p}^{\prime \prime}\right)$ соответственно. Следуюшее предложение немедленно вытекает из предложения 6.3.

ПРЕДЛОЖенИЕ 6.4. Концы $X$ и $Y$ линии Симпсона L могут быть вычислены по следующим формулам:

$$
\begin{aligned}
& X=\frac{1}{\omega(e)} \sum_{p=1}^{k^{\prime}} \omega\left(e_{p}^{\prime}\right) m_{p}^{\prime} e^{-i t_{p}^{\prime} \pi / 3} \\
& Y=\frac{1}{\omega(e)} \sum_{p=1}^{k^{\prime \prime}} \omega\left(e_{p}^{\prime \prime}\right) m_{p}^{\prime \prime} e^{-i t_{p}^{\prime \prime} \pi / 3}
\end{aligned}
$$


6.5. Вектор Максвелла. Пусть $\Gamma: G \rightarrow \mathbb{R}^{2}$ - произвольная взвешенная минимальная сеть, $G_{1}, \ldots, G_{m}$ - невырожденные компоненты графа $G$ и $\Gamma_{q}=\left.\Gamma\right|_{G_{q}}-$ соответствуюшие невырожденные компоненты сети $\Gamma$. Рассмотрим некоторую невырожденную компоненту $\Gamma_{q}$. Для каждой граничной вершины $v_{p}$ из $G_{q}$ обозначим через $\nu_{p}$ единичный вектор направления единственного входящего в вершину $\Gamma_{q}: v_{p} \rightarrow \mathbb{R}^{2}$ ребра $\Gamma_{q}: e_{p} \rightarrow \mathbb{R}^{2}$ сети $\Gamma_{q}$.

Каждому вектору $\nu_{p}$ соответствует комплексное число $e^{i \varphi_{p}}$. Далее, пусть, как и выше, $m_{p}$ - комплексное число, соответствующее точке $\Gamma\left(v_{p}\right)$. Обозначим через $\mu\left(\Gamma_{q}\right)$ комплексное число $\sum_{p} m_{p} \omega\left(e_{p}\right) \exp \left(-i \varphi_{p}\right)$, где сумма берется по всем граничным вершинам $v_{p}$ графа $G_{q}$.

ОПРЕДЕЛЕНИЕ. Комплексный вектор $\mu(\Gamma)=\left(\mu\left(\Gamma_{1}\right), \ldots, \mu\left(\Gamma_{m}\right)\right)$ называется вектором Максвелла взвешенной минимальной сети $Г$. Если $m=1$, то вектор Максвелла будем называть иислом Максвелла.

ПРЕДЛОЖЕНИЕ 6.5. Компонента $\mu\left(\Gamma_{q}\right)$ вектора Максвелла $\mu(\Gamma)$ взвешенной минимальной сети Штейнера $Г$ - это положстельное вещественное число, равное взвешенной длине невырожденной компоненты $\Gamma_{q}$ сети Г.

ДокАЗАТЕльство. Так как число Максвелла $\mu\left(\Gamma_{q}\right)$ взвешенной минимальной сети $\Gamma_{q}$ совпадает с $q$-й компонентой вектора Максвелла $\mu(\Gamma)$ сети $\Gamma$, предложение достаточно доказать в предположении, что сеть $Г$ невырождена.

Для каждой подвижной вершины $v \in G$ рассмотрим единичные векторы $n_{v}^{j}$ направлений входящих в вершину $\Gamma: v \rightarrow \mathbb{R}^{2}$ ребер. Так как сеть минимальна, то при каждом фиксированном $v$ линейная комбинация векторов $n_{v}^{j}$ с коэффициентами весами соответствуюших ребер - равна нулю. Поэтому равно нулю и произведение комплексного числа $\Gamma(v)$ на комплексное число, сопряженное к линейной комбинации соответствуюших векторам $n_{v}^{j}$ комплексных чисел с коэффищиентами - весами входящих в $v$ ребер. Следовательно, добавив эти равные нулю произведения к $\mu(\Gamma)$, мы не изменим значение $\mu(\Gamma)$.

Прибавим все эти (нулевые) произведения к $\mu(\Gamma)$. В полученной сумме мы изменим порядок суммирования, сгрупшировав вместе слагаемые, соответствуюшие одному и тому же ребру $e$, а затем уже просуммировав по всем ребрам из $G$. А именно, пусть $e$ - произвольное ребро из $G, A_{e}$ и $B_{e}$ - (комплексные) координаты концевых точек отрезка $\Gamma(e)$, а $n_{A}$ и $n_{B}$ - направление отрезка $\Gamma(e)$, ориентированного соответственно в сторону $A_{e}$ и $B_{e}$. Иными словами, $n_{A}=-n_{B}=B_{e} A_{e} /\left|B_{e} A_{e}\right|$. Пусть векторам $n_{A}$ и $n_{B}$ соответствуют комплексные числа $e^{i \varphi_{A}}$ и $e^{i \varphi_{B}}$, где $\varphi_{B}=$ $\pi+\varphi_{A}$. Тогда ребру $e$ в нашей сумме соответствует следуюшее выражение:

$$
\omega(e) e^{-i \varphi_{A}} A_{e}+\omega(e) e^{-i \varphi_{B}} B_{e}=\omega(e) e^{-i \varphi_{A}}\left(A_{e}-B_{e}\right) .
$$

В силу того, что векторы $n_{A}$ и $B_{e} A_{e}$ коллинеарны, рассматриваемое выражение равно взвешенной длине $\omega(e)|A B|$ ребра $\Gamma: e \rightarrow \mathbb{R}^{2}$. Поэтому $\mu(\Gamma)$ равно взвешенной длине сети $\Gamma$, что и требовалось доказать. Доказательство предложения закончено.

ЗАмечАНИЕ. Отметим, что мнимая часть каждой компоненты $\mu\left(\Gamma_{i}\right)$ вектора Максвелла совпадает по определению с внешней формой Максвелла $\mu\left(\Gamma_{i}\right)$ степени нуль, определенной выше.

Из предложения 6.5 немедленно вытекает следуюший результат. 
ПРЕДЛОЖЕНИЕ 6.6. Пусть Г и $\Gamma^{\prime}-$ две параллельные взвешенные минимальные сети с одной и той же граничей $\varphi$. Тогда векторы Максвелла сетей Г и Г' совпадают. В частности, как соответствующие компоненть сетей $Г$ и $\Gamma^{\prime}$, так и сами сети $\Gamma$ и $\Gamma^{\prime}$ имеют одинаковую взвешенную длину.

Пусть $G$ - невырожденный взвешенный граф Штейнера с эффективной границей $\partial G$ и весовой функцией $\omega$, а $\varphi: \partial G \rightarrow \mathbb{R}^{2}-$ некоторое граничное отображение. Пусть $\Gamma: G \rightarrow \mathbb{R}^{2}$ - взвешенная минимальная сеть с границей $\partial \Gamma=\varphi$. Выберем произвольную граничную вершину $v_{0}$ графа $G$, и пусть $v_{1}, \ldots, v_{n}$ - остальные граничные вершины графа $G$, а $e_{p}$ - единственное ребро из $G$, инцидентное $v_{p}$. Положим $m_{p}=\Gamma\left(v_{p}\right)$. Пусть $t_{p}-$ взвешенное число вращения от ребра $e_{0}$ к ребру $e_{p}$, индуцированное сетью Г. Определим число $\operatorname{Mаксвелла~} \mu\left(\Gamma, e_{0}\right)$ napы $\left(\Gamma, e_{0}\right)$ так:

$$
\mu\left(\Gamma, e_{0}\right)=\sum_{p=1}^{n} m_{p} \omega\left(e_{p}\right) e^{-i t_{p} \frac{\pi}{3}}-m_{0} \omega\left(e_{0}\right)
$$

ПРЕДЛОЖЕНИЕ 6.7. Пусть $\Gamma^{\prime} u \Gamma^{\prime \prime}-\partial в е$ (ориентируемо) планарно әквивалентные невыроэсденные взвешенные минимальные сети топологии G. Тогда эти сети параллельны, если и только если $\arg \left(\mu\left(\Gamma^{\prime}, e\right)\right)=\arg \left(\mu\left(\Gamma^{\prime \prime}, e\right)\right)$ для некоторого (а значит, и произвольного) граничного ребра е графа $G$.

ДокАЗАТЕльство. Действительно, пусть $v$-произвольная граничная вершина графа $G$ и $e$ - единственное инцидентное ей ребро из $G$. Обозначим через $e^{i \alpha}$ направление вектора $\Gamma^{\prime}(e)$, ориентированного от точки $\Gamma^{\prime}(v)$. Тогда достаточно заметить, что значения чисел Максвелла $\mu\left(\Gamma^{\prime}\right)$ и $\mu\left(\Gamma^{\prime}, e\right)$ связаны так: $\mu\left(\Gamma^{\prime}\right)=$ $\mu\left(\Gamma^{\prime}, e\right) e^{-i \alpha}$. Аналогично, если $e^{i \beta}$ - направление вектора $\Gamma^{\prime \prime}(e)$, ориентированного от точки $\Gamma^{\prime \prime}(v)$, то $\mu\left(\Gamma^{\prime \prime}\right)=\mu\left(\Gamma^{\prime \prime}, e\right) e^{-i \beta}$. Однако $\mu\left(\Gamma^{\prime}\right)$ и $\mu\left(\Gamma^{\prime \prime}\right)-$ положительные вещественные числа (см. предложение 5.8), поэтому параллельность сетей $\Gamma^{\prime}$ и $\Gamma^{\prime \prime}$, равносильная сонаправленности векторов $\Gamma^{\prime}(e)$ и $\Gamma^{\prime \prime}(e)$, эквивалентна равенству аргументов чисел Максвелла $\mu\left(\Gamma^{\prime}, e\right)$ и $\mu\left(\Gamma^{\prime \prime}, e\right)$.

6.6. Построение деформации невырожденной минимальной сети. Пусть $G$ - невырожденный взвешенный граф Штейнера с эффективной гранищей $\partial G$ и весовой функцией $\omega$ и $\varphi$ - некоторое граничное отображение. Предположим, что существует взвешенная минимальная сеть $\Gamma \in[G, \varphi]$. Пусть $e_{1}, \ldots, e_{k}-$ ребра графа $G$ и $A_{1}, \ldots, A_{k}$ - внутренние точки этих ребер, $A_{p} \in e_{p}$, такие, что топологический граф $G^{\prime}$, полученный из $G$ разрезанием ребер $e_{p}$ по точкам $A_{p}$, является 2 -деревом. Ясно, что $k$ равно цикломатическому числу графа $G$. Отметим, что в силу сделанных предположений все ребра $e_{i}$ - внутренние циклические ребра графа $G$. Обозначим через $\Gamma^{\prime}$ сеть, полученную из сети $\Gamma$ разрезанием по всем точкам $A_{p}$. Пусть $\partial G^{\prime}-$ эффективная граница дерева $G^{\prime}$. Ясно, что $\partial G^{\prime}$ получается из $\partial G$ добавлением вершин $A_{p}^{1}$ и $A_{p}^{2}$ дерева $G^{\prime}$, на которые распались точки $A_{p}$. Обозначим через $\partial \Gamma^{\prime}$ эффективную границу сети $\Gamma^{\prime}$.

Рассмотрим граничное отображение $\varphi^{\prime}$ дерева $G^{\prime}$, полученное из $\partial \Gamma^{\prime}$ мальм смещением точек $\Gamma\left(A_{p}\right)$ в направлениях, перпендикулярных ребрам $\Gamma\left(e_{p}\right)$. Иными словами, если $B_{p}$ - результат такого смещения для точки $\Gamma\left(A_{p}\right)$, то положим $\varphi^{\prime}\left(A_{p}^{1}\right)=\varphi^{\prime}\left(A_{p}^{2}\right)=B_{p}$. Пусть $e_{p}^{1}$ и $e_{p}^{2}$ - граничные ребра из $G^{\prime}$, на которые распалось ребро $e_{p} \subset G$ после выбрасывания точки $A_{p}$. Ориентируем их в сторону граничных вершин $A_{p}^{1}$ и $A_{p}^{2}$ соответственно. По предложению 6.1 для достаточно 
малых смешений сушествует взвешенная минимальная реализация $\bar{\Gamma}^{\prime}$ дерева $G^{\prime}$ с границей $\varphi^{\prime}$ и эта реализация непрерывно зависит от граничного отображения $\varphi^{\prime}$. Отсюда вытекает, что оснашение врашения сети $\bar{\Gamma}^{\prime}$ совпадает с оснашением вращения сети $\Gamma^{\prime}$. Поэтому, в частности, в силу предложения 5.1 при таких взвешенных минимальных реализациях образы ориентированных ребер $e_{p}^{1}$ и $e_{p}^{2}$ остаются противоположно направленными. Поэтому, склеив сеть $\bar{\Gamma}^{\prime}$ по каждой паре вершин $\varphi^{\prime}: A_{p}^{1} \rightarrow \mathbb{R}^{2}$ и $\varphi^{\prime}: A_{p}^{2} \rightarrow \mathbb{R}^{2}$, получим новую взвешенную минимальную реализацию графа $G$ с границей $\partial \Gamma$.

Пусть $n_{p}$ - единичная нормаль к $\Gamma\left(e_{p}\right)$ в точке $\Gamma\left(A_{p}\right)$. Тогда смешение точки $\Gamma\left(A_{p}\right)$ задается числом $t_{p}$ таким, что $B_{p}=\Gamma\left(A_{p}\right)+t_{p} n_{p}$. Поэтому построенная вьше сеть $\bar{\Gamma}^{\prime}$ однозначно определяется точкой $t=\left(t_{1}, \ldots, t_{k}\right)$ из $\mathbb{R}^{k}$, причем ясно, что разным точкам $t$ соответствуют разные такие сети. Кроме того, по предложению 5.6 каждому малому $t$ соответствует ровно одна сеть $\bar{\Gamma}^{\prime}$, которую мы обозначим через $\bar{\Gamma}_{t}^{\prime}$. Наконец, как и выше, для каждой сети $\bar{\Gamma}_{t}^{\prime}$ мы построим свою взвешенную минимальную реализацию $\Gamma_{t}$ графа $G$ с границей $\partial \Gamma$. Таким образом, мы построили $k$-параметрические вариации сетей $\bar{\Gamma}^{\prime}$ и $\Gamma$, являющиеся топологическими вложениями $\nu^{\prime}$ и $\nu$ малого открытого $k$-мерного куба $I^{k}$ из $\mathbb{R}^{k}$ с центром в нуле в пространства $\mathfrak{L}\left(G^{\prime}\right)$ и $[G, \varphi]$ соответственно.

Как и в случае обычных локально минимальных сетей, отображения $\nu$ и $\nu^{\prime}$ обладают дополнительно следуюшими важными свойствами (доказательство дословно повторяет соответствуюшие доказательства из [47]).

ПРЕДЛОЖЕНИЕ 6.8. Отображсение $\nu$ переводит куб $I^{k}$ взаимно однозначно на некоторую окрестность сети $\Gamma$ во множестве $[G, \varphi]_{\min }$.

Отображения $\nu$ и $\nu^{\prime}$ аффинны.

Итак, отображения $\nu$ и $\nu^{\prime}$ - аффинные отображения из некоторого $k$-мерного куба $I^{k} \subset \mathbb{R}^{k}$ в пространства $[G, \varphi]$ и $\mathfrak{L}\left(G^{\prime}\right)$ соответственно. Из взаимной однозначности этих отображений вытекает, что их образы - это некоторые $k$-мерные кубы. Продолжим эти отображения по аффинности на все пространство $\mathbb{R}^{k}$ и полученные отображения обозначим через $\bar{\nu}$ и $\bar{\nu}^{\prime}$ соответственно. Понятно, что $\nu\left(I^{k}\right)$ и $\nu^{\prime}\left(I^{k}\right)$ - открытые подмножества в $\bar{\nu}\left(\mathbb{R}^{k}\right)$ и $\bar{\nu}^{\prime}\left(\mathbb{R}^{k}\right)$ соответственно.

УТВеРЖДЕНИЕ 6.2. Множество $[G, \varphi]_{\min }$ челиком лежит в $\bar{\nu}\left(\mathbb{R}^{k}\right)$ и является выпуклыц $k$-мерным телом в $\bar{\nu}\left(\mathbb{R}^{k}\right)$. Более того, каждая взвешенная минимальная сеть $\Gamma$ топологии $G$ с гранищей $\partial \Gamma=\varphi$ является внутренней точкой выпуклого тела $[G, \varphi]_{\min } \subset \bar{\nu}\left(\mathbb{R}^{k}\right)$.

Из приведенных выше утверждений непосредственно получается следуюший результат.

Теорема 6.1. Пусть Г-не имеющая слабо фиктивных вериин степени 3 взвешенная минимальная сеть Штейнера типа $G$ в $\mathbb{R}^{2}$ с границей $\varphi$ и весовой функцией $\omega$. Обозначим через $k$ чикломатическое число подвижного подграфа $\widehat{G}_{\varphi}$ графа $G$, через $f$ - количество фиктивных вершин сети $\Gamma$, через $m-$ количество ребер сети $\Gamma$, а через $[G, \varphi]$ - конфигурачионное пространство подвижных вериин графа $G$.

Тогда множество взвешенных минимальных сетей типа $G$ с границей $\varphi$ представляет собой внутренность выпуклого $(k+f)$-мерного многогранника 
$[G, \varphi]_{\min }$, являющегося множеством всех минимумов функиии взвешенной длинь $\ell_{\omega}:[G, \varphi] \rightarrow \mathbb{R}$. Многогранник $[G, \varphi]_{\min }$ имеет не более чем $m$ граней максимальной размерности.

Все минимальнье сети данной топологии и с данной границей параллельнь между собой и имеют одинаковую взвешенную длину. Локус $\operatorname{loc}_{\varphi}(v)$ произвольной вериинь $v$ из $G$ имеет следующий вид. Если $v$-вершина второго уровня, то $\operatorname{loc}_{\varphi}(v)$ состоит из одной точки; если $v$ - вериина первого уровня,

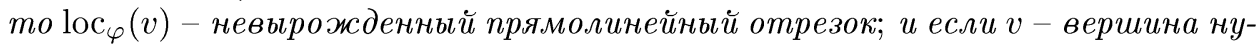
левого уровня, то $\operatorname{loc}_{\varphi}(v)$ - невырожсенный выпуклый многоугольник, число вериин которого не превосходит $\mathrm{m}$.

Приведем здесь одно нетривиальное следствие этой теоремы.

СлЕДСТВИЕ 6.1. В обозначениях теоремы 6.1 если $k=2 u f=0$, m.е. иикломатическое число подвижного подграфа графа $G$ равно 2 , и граф $G$ вообще не содержит слабо фиктивных (и, в частности, фиктивных) вериин, то локусы всех вериин нулевого уровня - выпуклые многоугольники с одинаковым числом сторон.

ДокАЗАТЕЛьСтво. Действительно, в этом случае многогранник $[G, \varphi]_{\min }$ имеет размерность 2. Так как локус вершины второго уровня является в силу теоремы 6.1 невырожденным многоугольником, а по следствию 4.2 - проекцией многогранника $[G, \varphi]_{\min }$, то локус имеет столько же вершин, сколько и многоугольник $[G, \varphi]_{\min }$. Доказательство закончено.

\section{Список литературы}

1. Clark R. C. Communication networks, soap films and vectors // Phys. Ed. 1981. V. 16. P. $32-37$.

2. Dijkstra E. W. A note on two problems with connection with graphs // Numer. Math. 1959. V. 1. № 5. P. 269-271.

3. Du D.Z., Hwang F. K. A New Bound for the Steiner Ratio // Trans. Amer. Math. Soc. 1983. V. 278. № 1. P. 137-148.

4. Du D.Z., Hwang F.K. A Proof of Gilbert-Pollak's Conjecture on the Steiner Ratio. Preprint DIMACS Technical Report. 1990. № 90-72.

5. Du D. Z., Hwang F. K. An approach for proving lower bounds: solution of Gilbert-Pollak's Conjecture on the Steiner Ratio // Proc of the 31st annual symp. on found. of comp. science, 1990 .

6. Du D.Z., Hwang F. K., Chao S. C. Steiner minimal trees for points on a circle // Proc. Amer. Math. Soc. 1985. V. 95. № 4. P. 613-618.

7. Du D.Z., Hwang F.K., Weng J.F. Steiner minimal trees for points on a zig-zag lines // Trans. Amer. Math. Soc. 1983. V. 278. № 1. P. 149-156.

8. Du D. Z., Hwang F. K., Weng J. F. Steiner minimal trees for Regular Polygons // Disc. and Comp. Geometry. 1987. V. 2. P. 65-84.

9. Du D.Z., Hwang F.K., Yao E.N. The Steiner ratio conjecture is true for five points // J. Combin Th. Ser. A38. 1985. P. 230-240.

10. Du D.Z., Hwang F.K., Song G.D., Ting G. T. Steiner minimal trees on sets of four points // Discr. and Comp. Geometry. 1987. V. 2. P. 401-414.

11. Garey M.R., Johnson D.S. The complexity of computing Steiner minimal trees // SIAM J. Algebraic Discrete Methods. 1977. V. 32. P. 835-859.

12. Garey M.R., Graham R.L., Johnson D.S. Some NP-complete geometric problems Eighth Annual Symp. on Theory of Comput. 1976. P. 10-22.

13. Gilbert E. N., Pollak H. O. Steiner minimal trees // SIAM J. Appl. Math. 1968. V. 16. № 1. P. 1-29. 
14. Hildebrandt S., Tromba A. Mathematics and optimal form. New York: An imprint of Scientific American Books, Inc., 1984.

15. Hwang F. K. A linear time algorithm for full Steiner trees // Oper. Res. Letter. 1986. V. 5. P. 235-237.

16. Hwang F.K., Weng J.F. Hexagonal coordinate Systems and Steiner minimal trees // Disc. Math. 1986. V. 62. P. 49-57.

17. Hwang F. K., Richards D., Winter P. The Steiners Tree Problem. Elsevier Science Publishers, 1992.

18. Jarnik V., Kössler M. O minimalnich grafeth obeahujicich n danijch bodu // Cas. Pest. Mat. a Fys. 1934. V. 63. P. 223-235.

19. Kruskal J.B. On the shortest spanning subtree of a graph and traveling salesman problem // Proc. Amer. Math. Soc. 1956. V. 7. P. 48-50.

20. Melzak Z. A. On the problem of Steiner // Canad. Math. Bull. 1960. V. 4. P. 143-148.

21. Melzak Z. A. Companion to concrete mathematics. N. Y.: Wiley-Interscience, 1973.

22. Pollak H. O. Some remarks on the Steiner problem // J. Combin. Thy. Ser. A24. 1978. P. 278-295.

23. Preparata F., Shamos M. Computational Geometry. An introduction. N. Y.: Springer-Verlag, 1985.

24. Prim R. C. Shortest connecting networks and some generalizations // BSTJ. 1957. V. 36. P. 1389-1401.

25. Rubinstein J.H., Thomas D.A. The Steiner ratio conjecture for six points // J. Combin. Thy. Ser. A58. 1989. P. 54-77.

26. Rubinstein J.H., Thomas D. A. Steiner Minimal Networks for Convex Configurations. Preprint / Univ. Melbourne. Dep. of Math. series № $15-1991$.

27. Rubinstein J. H., Thomas D. A. Graham's Problem On Shortest Networks for Points on a Circle // Algorithmica. 1992. V. 7. P. 193-218.

28. Shamos M. I. Computational Geometry. Ph.D. Thesis. Dept. of Comput. Sci., Yale Univ., 1978.

29. Smith W. D. How to find Steiner minimal trees in Euclidean $d$-space // Algorithmica. 1992. № 7. P. 137-177.

30. Емеличев В. А. и др. Лекции по теории графов. М.: Наука, 1990.

31. Дао Чонг Тхи, Фоменко А. Т. Минимальные поверхности и проблема Плато. М.: Наука, 1987.

32. Фоменко А. Т. Топологические вариационные задачи. М.: Изд-во МГУ, 1984.

33. Фоменко А. Т. Вариационные методы в топологии. М.: Наука, 1982.

34. Тужилин A.A., Фоменко A. Т. Элементы геометрии и топологии минимальных поверхностей. М.: Наука, 1991.

35. Иванов А. О. Геометрия плоских локально минимальных бинарных деревьев // Матем. сб. 1995. Т. 186. № 9. С. 45-76.

36. Иванов А. О. Плоские взвешенные минимальные бинарные деревья // Фундаментальные проблемы матем. 1996. Т. 2. № 2. С. 511-562.

37. Иванов A.О., Тужилин A.A. Решение задачи Штейнера для выпуклых границ // УМH. 1990. Т. 45. № 2. С. 207-208.

38. Иванов A. О., Тужилин А. А. Задача Штейнера для выпуклых границ или плоские минимальные сети // Матем. сб. 1991. Т. 182. № 12. С. 1813-1844.

39. Иванов A. O., Тужилин A. А. Геометрия минимальных сетей и одномерная проблема Плато // УМН. 1992. Т. 47. № 2. С. 53-115.

40. Ivanov A.O., Tuzhilin A. A. The Steiner problem for convex boundaries. I: general case // Advances in Soviet Mathematics. 1993. V. 15. P. 15-92.

41. Ivanov A.O., Tuzhilin A. A. The Steiner problem for convex boundaries. II: the regular case // Advances in Soviet Mathematics. 1993. V. 15. P. 93-131.

42. Ivanov A. O., Tuzhilin A. A. Minimal Networks. The Steiner Problem and Its Generalizations. Boca Raton: CRC Press, 1994.

43. Иванов A. О., Тужилин A. А. Топологии локально минимальных плоских бинарных деревьев // УМН. 1994. Т. 49. №6. С. 191-192. 
44. Иванов А. О., Тужилин А. А. Взвешенные минимальные 2-деревья // УМН. 1995. T. 50. № 3. C. $155-156$.

45. Иванов А. О., Тужилин А. А. Геометрия плоских линейных деревьев // УМН. 1996. T. 51. № 2. C. 161-162.

46. Иванов А. О., Тужилин А.А. Число вращения плоских линейных деревьев // Матем. сб. 1996. Т. 187. № 8. С. 41-92.

47. Иванов А. О., Тужилин A. А. Геометрия множества минимальных сетей данной топологии с фиксированной границей // Изв. РАН. Сер. матем. 1997. Т. 61. № 6. С. 119-152.

48. Иванов А.О., Исхаков И.В., Тужилин А.А. Минимальные сети на правильных многоугольниках: реализация линейных паркетов // Вест. МГУ. Сер. матем. 1993. №6 . C. $77-81$.

49. Иванов А. О., Птищьна И. В., Тужилин А. А. Классификация замкнутых минимальных сетей на плоских двумерных торах // Матем. сб. 1992. Т. 183. № 12. С. 3-44.

50. Тужилин $A$. A. Минимальные бинарные деревья с правильной границей: случай скелетов с четырьмя концами // Матем. сб. 1996. Т. 187. № 4. С. 117-159.

51. Тужилин $A$. A. Минимальные бинарные деревья с правильной границей: случай скелетов с пятью концами // Матем. заметки. 1997. Т. 61. №6. С. 907-921.

52. Kuhn H. W. Steiner's problem revisted // Studies in Optimization. Ser. Studies in Math. V. 10 / Math. Assoc. Amer. G. B. Dantzig, B. C. Eaves. 1975. P. 53-70.

53. Zacharias M. Encyklopädie der Mathematischen. Wissenschaften. V. III. AB9.

Поступило в редакцию

17.II.1998 